\title{
1 Spatial single-cell profiling of intracellular metabolomes in situ
}

2 Luca Rappez ${ }^{1,2}$, Mira Stadler ${ }^{3}$, Sergio Triana ${ }^{1,2}$, Prasad Phapale ${ }^{4}$, Mathias Heikenwalder ${ }^{3}$,

3 Theodore Alexandrov ${ }^{1,4,5, *}$

$4{ }^{1}$ Structural and Computational Biology Unit, European Molecular Biology Laboratory (EMBL),

5 Heidelberg, 69117 Germany; ${ }^{2}$ Collaboration for joint PhD degree between EMBL and Heidelberg

6 University, Faculty of Biosciences, Germany; ${ }^{3}$ Institute of Chronic Inflammation and Cancer, Deutsches

7 Krebs-Forschungszentrum (DKFZ), 69120 Heidelberg, Germany; ${ }^{4}$ Metabolomics Core Facility, EMBL,

8 Heidelberg, 69117 Germany; ${ }^{5}$ Skaggs School of Pharmacy and Pharmaceutical Sciences, University of

9 California San Diego, CA 92093, La Jolla, USA

10 * Correspondence: theodore.alexandrov@embl.de

11

12

13 


\section{Summary}

15 The recently unveiled extent of cellular heterogeneity demands for single-cell investigations of

16 intracellular metabolomes to reveal their roles in intracellular processes, molecular

17 microenvironment and cell-cell interactions. To address this, we developed SpaceM, a method

18 for in situ spatial single-cell metabolomics of cell monolayers which detects $>100$ metabolites in

$19>10000$ individual cells together with fluorescence and morpho-spatial cellular features. We

20 discovered that the intracellular metabolomes of co-cultured human HeLa cells and mouse

21 NIH3T3 fibroblasts predict the cell type with $90.4 \%$ accuracy and revealed a short-distance

22 metabolic intermixing between HeLa and NIH3T3. We characterized lipid classes composing

23 lipid droplets in steatotic differentiated human hepatocytes, and discovered a preferential

24 accumulation of long-chain phospholipids, a co-regulation of oleic and linoleic acids, and an

25 association of phosphatidylinositol monophosphate with high cell-cell contact. SpaceM provides

26 single-cell metabolic, phenotypic, and spatial information and enables spatio-molecular

27 investigations of intracellular metabolomes in a variety of cellular models.

\section{Keywords}

30 Spatial single-cell metabolomics, imaging mass spectrometry, microscopy, heterogeneity,

31 metabolic intermixing, macrovesicular steatosis, lipid droplets, cell-cell contact, SpaceM 


\section{Introduction}

34 Multicellular organisms contain a multitude of cells of distinct and diverse functions,

35 morphologies, and molecular compositions. Each single cell has a unique intracellular

36 metabolome, a dynamic repertoire of metabolites and lipids involved in virtually all cellular

37 processes. Aside with metabolites and lipids serving as building blocks and energy sources

38 within the cell, recent discoveries unveiled their roles in signaling (Wellen and Thompson,

39 2012), epigenome regulation (Sharma and Rando, 2017), immunity (Buck et al., 2017),

40 inflammation (Murphy and O’Neill, 2018), host-microbe interactions (Sharon et al., 2014), and

41 cancer (Pavlova and Thompson, 2016). At the same time, the progress of single-cell technologies

42 revealed the extent and biological functions of cellular heterogeneity (Altschuler and $\mathrm{Wu}, 2010$ )

43 within tissues, organs (Marioni and Arendt, 2017), tumors (Patel et al., 2014), and even among

44 monoclonal cells in culture (Lee et al., 2014; Pelkmans, 2012; Russell et al., 2018). The

45 discovered critical roles of metabolism and the growing awareness of a hidden world beneath

46 population averages created an urgent need to investigate intracellular metabolism at the single-

47 cell level (Rubakhin et al., 2013; Zenobi, 2013). In the recent years, the sensitivity of mass

48 spectrometry-based metabolite detection has improved substantially opening novel avenues to

49 metabolomics of either single cells or small groups of cells (Do et al., 2017; Guillaume-Gentil et

50 al., 2017; Ibáñez et al., 2013; Merrill et al., 2017) and even at a subcellular level (Passarelli et al.,

51 2017). However, despite these methods successfully demonstrated detection of metabolites in

52 individual cells, analytical and computational challenges precluded studies of spatio-molecular

53 organization and cellular heterogeneity, and prevented discovering the links between

54 intracellular metabolomes, cellular phenotypes and spatial organisation of cells. 
55 To bridge this gap, we designed SpaceM, a method for spatial single-cell metabolomics of cell

56 monolayers that integrates MALDI-imaging mass spectrometry with bright-field and

57 fluorescence microscopy. Integration with microscopy enables associating metabolites with

58 fluorescence and morphological cell properties (fluorescent reporter intensity, area, compactness,

59 shape) as well as with spatial features quantifying multi-cellular organization. The integration

60 was enabled by a method for precise detection of parts of cells sampled by MALDI laser with the

61 help of sequential microscopy, novel image analysis, and a novel cell-ablation marks

62 normalisation strategy. Using the False Discovery Rate-controlled metabolite annotation, and

63 novel methods for unbiased selection of intracellular metabolites and for filtering out poor

64 quality cells allowed us to perform high-throughput analyses with $>100$ metabolites detected in

$65>10000$ individual cells, with a high reproducibility between replicates. We validated SpaceM by

66 investigating metabolomes of co-cultured HeLa and mouse fibroblasts cells as well as of

67 differentiated human steatotic hepatocytes stimulated with pro-inflammatory factors that

68 provided rich metabolic, phenotypic, and spatial information. 


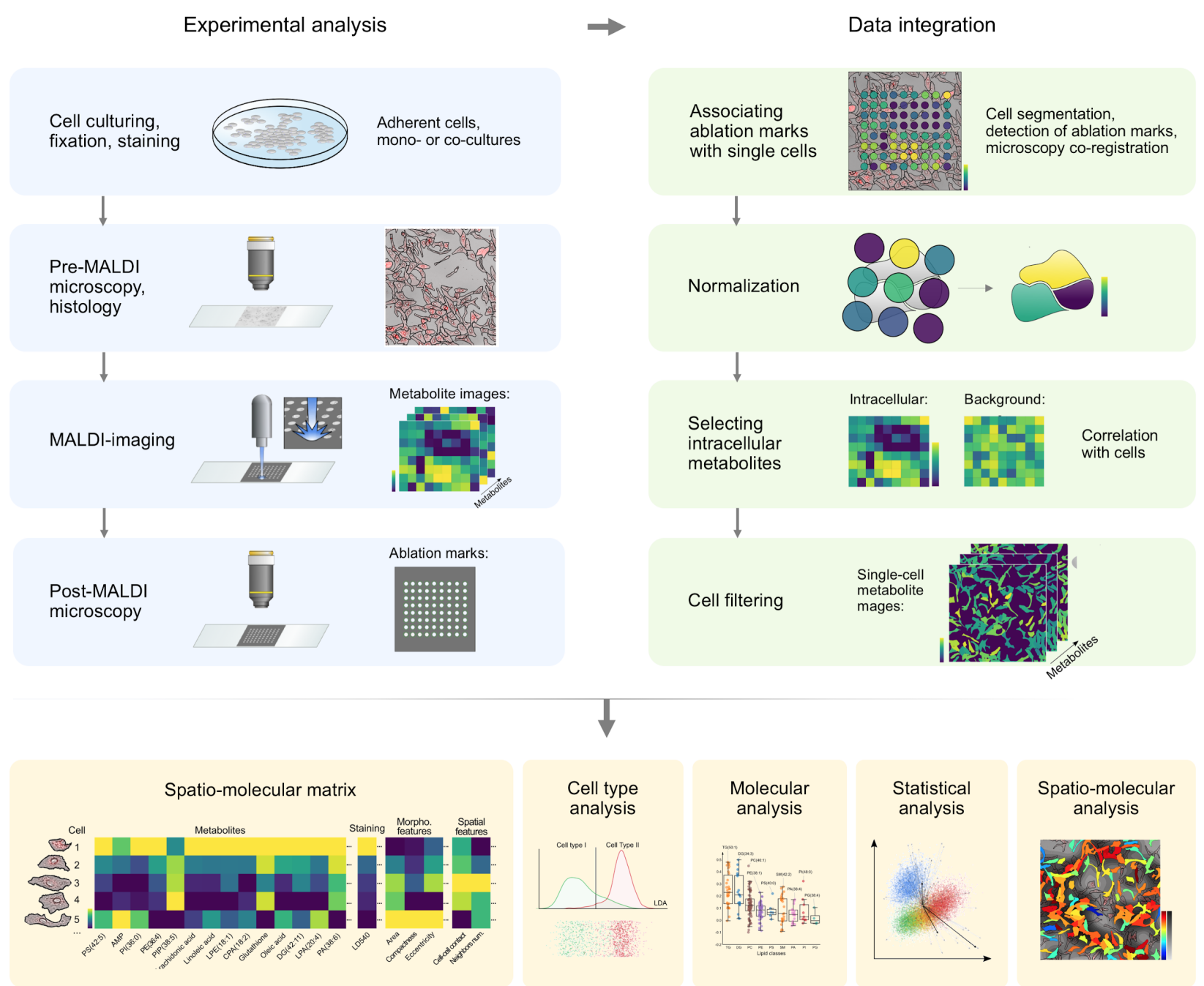

Figure 1. SpaceM method for spatial single-cell metabolomics of cell monolayers by

71 integrative microscopy and MALDI imaging mass spectrometry. The experimental part of the

72 workflow includes cell culturing, pre-MALDI and post-MALDI microscopy and histology, and

73 MALDI imaging mass spectrometry. The data integration part includes associating of MALDI

74 laser ablation marks with individual cells, strategies for normalization, selecting intracellular

75 metabolites, and cell filtering (see Figure S1 for a detailed workflow). SpaceM outputs a single-

76 cell spatio-molecular matrix providing rich information for a variety of analyses, in particular to

77 characterise cell types, associate single-cell metabolomes with a fluorescent phenotype, 

molecular associations.

81 Results

\section{The SpaceM method}

83 SpaceM relies on using Matrix Assisted Laser Desorption Ionization (MALDI)-imaging mass

84 spectrometry, a spatially-resolved mass spectrometry technology for detection of a wide range of 85 molecules (Baker et al., 2017). MALDI-imaging is increasingly used for spatial metabolomics

86 (Palmer et al., 2016) and was demonstrated to achieve the femtomolar-levels sensitivity

87 (Soltwisch et al., 2015). This, together with soft ionisation preventing excessive in-source

88 molecular fragmentation makes it a perfect choice for single-cell metabolomics as demonstrated

89 by others (Do et al., 2017; Ibáñez et al., 2013). The experimental part of SpaceM combines

90 MALDI-imaging with microscopy as well as with collecting supporting information to integrate

91 these two sources of data (Figure 1; for a detailed workflow see Figure S1). The cells for

92 SpaceM are cultured on a labtek chamber glass slide in a monolayer, with the cell confluence

93 sufficient to allow cells to interact between each other but at the same time preventing the growth

94 of cells on top of each other. After washing, cells are fixed to halt enzymatic activity, stained

95 with a fluorescent dye with the staining protocol compatible with metabolomics, and dried in a

96 desiccator following regular cell preparation protocols. SpaceM requires the Hoechst (or any

97 similar) staining for nuclei detection. For investigation of the steatotic hepatocytes, we also used

98 the lipophilic LD540 staining to detect lipid droplets (Spandl et al., 2009). Then, bright-field and

99 fluorescence microscopy images of cells are collected with the following two aims in mind. 
100 First, the cell segmentation of the microscopy images provides cell outlines and enables cell

101 localization. Second, microscopy provides rich phenotypic information about single-cell

102 fluorescence, immunochemistry and spatio-morphological properties of the cells. In the next

103 experimental step, MALDI-imaging is applied to the dried cells to collect mass spectra across

104 cells and extracellular areas. MALDI-imaging procedure starts with application of an ionisation-

105 enhancing matrix. Similar to MALDI-imaging of tissues, we used a robotic sprayer for enhanced

106 extraction, high spatial resolution, and high reproducibility. MALDI-imaging generates big

107 datasets with millions of mass-to-charge channels. For finding metabolic signals in this data, we

108 exploited the False Discovery Rate-controlled metabolite annotation implemented as the

109 METASPACE cloud software (http://metaspace2020.eu) (Palmer et al., 2017). METASPACE is

110 an essential step as it reduces millions of mass-to-charge (m/z)-values to 100 metabolite

111 annotations, filters out signals representing matrix and contaminants, ensures quality control and

112 represents metabolite images a user-friendly way. In the last experimental step, we performed

113 post-MALDI microscopy to determine which cells were sampled by the MALDI-imaging laser

114 and to associate MALDI-imaging spectra with the cells. Next, we performed data integration

115 with the first step associating ablation marks with individual cells. We detected MALDI laser

116 ablation marks in post-MALDI microscopy images using a customized 2D Fourier

117 Transformation image analysis method that exploits similarities between ablation marks and the

118 regularity in spacings between them. Then, we obtained positions of the MALDI-imaging

119 ablation marks within the cell areas by co-registering pre-MALDI microscopy images

120 (containing cell outlines) with post-MALDI microscopy images (containing ablation marks

121 outlines) (Figure S2). For a majority of cells, a cell was sampled with just one ablation mark. In

122 our benchmarking experiment with HeLa cells and NIH3T3 fibroblast (described later), 72.25\%, 
$12323.8 \%, 2.8 \%, 0.9 \%$ cells were sampled with 1, 2, 3, 4 ablation marks, respectively. To integrate

124 metabolic profiles from several ablation marks co-sampling the same cell as well as to reduce the

125 confusion of co-sampled cells, we developed a cell-ablation marks normalization strategy

126 (Figure 2). The normalization provides the metabolite intensity normalized by the area that is a

127 natural readout for metabolite concentration. Next, we developed a strategy to distinguish

128 intracellular from extracellular metabolites by requiring an intracellular metabolite intensities to

129 be highly correlated with the cell spatial distribution. Finally, similar to cell filtration strategies

130 in single-cell RNA-seq (Ilicic et al., 2016), we filter out poor-quality cells with low numbers of

131 metabolite annotations with the cutoff determined as the 5\%-percentile of the numbers of

132 annotations for all cells in an experiment.
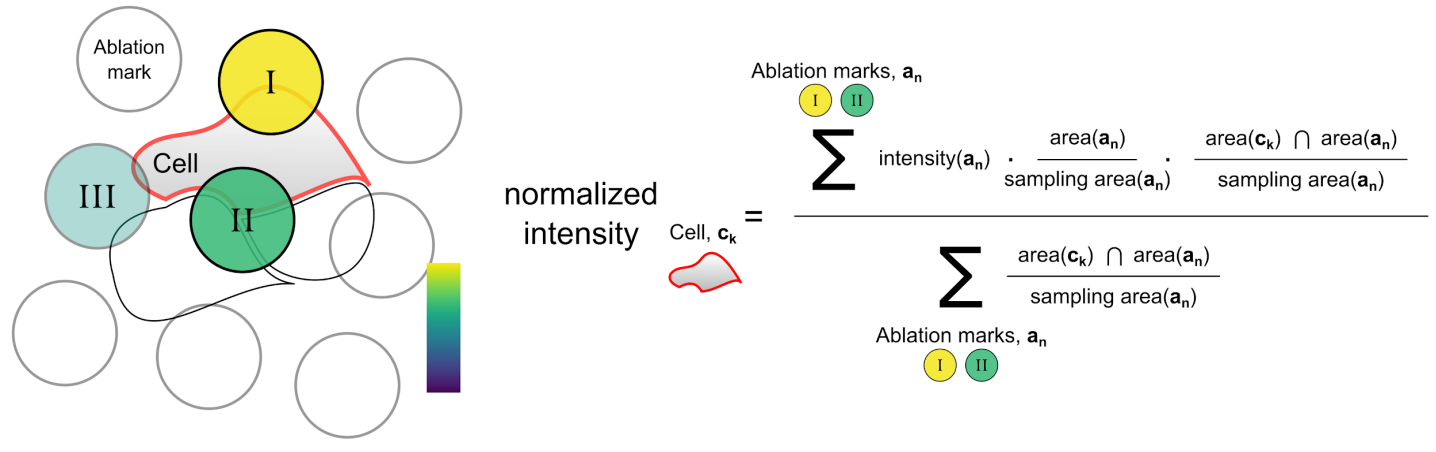

134 Figure 2. Normalization strategy for assigning metabolic intensities to individual cells. The

135 intensity assigned to a cell for a given metabolite is calculated as a weighted mean of the

136 metabolite intensities from the ablation marks sampling that cell. To increase the contribution of

137 ablation marks which mainly sample the cell of interest, the weight of each ablation mark is

138 proportional to the overlap of the ablation mark and the cell. To reduce the contribution of

139 ablation marks which mainly sample extracellular areas, the weight of each ablation mark is

140 reversely proportional to its extracellular sampling area. Ablation marks sampling

141 predominantly extracellular areas are filtered out (as the illustrated here ablation mark III). 
142 area $\left(a_{n}\right)$ stands for the area of ablation mark $a_{n}$; sampling area $\left(a_{n}\right)$ stands for the intracellular

143 area of ablation mark $a_{n}$; area $\left(c_{k}\right)$ stands for the area of cell $c_{k}$; all areas are computed in

144 microscopy pixels.

145 Ultimately, SpaceM provides a single-cell spatio-molecular matrix that, for each cell, comprises

146 a multiplex readout of the cell metabolic profile, fluorescence intensity, morphological features,

147 and spatial features (Figure 1). This information enables statistical analysis, phenotype-

148 metabolome correlation, and/or spatio-molecular interrogation of single cells in the genuine

149 spatial context.

\section{SpaceM predicts cell types with single-cell resolution}

151 For validation of the method, we evaluated whether SpaceM can predict the cell type of

152 spatially-heterogeneous co-cultured human HeLa cells and mouse NIH3T3 fibroblasts (Figure

153 3). We considered six samples (two replicates of co-cultures, and two replicates of control

154 monocultures for each cell type). HeLa and NIH3T3 cells constitutively expressed H2B-mCherry

155 and GFP, respectively, making them easily discernible by fluorescence microscopy. Automated

156 assignment of the cell type was done using a linear separating boundary between mCherry and

157 GFP fluorescence intensities (Figure 3A). Overall, metabolic profiles of 88 metabolites with an

$158 \mathrm{FDR} \leq 10 \%$ in at least one sample were obtained for 1624 cells in co-cultures (958 HeLa and 666

159 NIH3T3 cells) and 2197 cells in monocultures (1603 HeLa and 594 NIH3T3 cells). Support

160 Vector Machine classification of the single-cell metabolic profiles with the 10-fold cross-

161 validation for unbiased choice of the Gaussian kernel gamma predicted the cell type with $90.4 \%$

162 accuracy for the co-cultured cells. To evaluate the richness of the detected metabolic profiles, we

163 used only a random subset of all 88 metabolites and were able to consistently predict the cell 
164 type in co-cultures with $87.6 \%$ accuracy even when using only a half of all detected metabolites

165 (Figure 3B). This indicates the richness of the detected intracellular metabolomes and its

166 biological relevance in the context of cell type classification. Moreover, by applying the

167 statistical t-test we have identified the molecular markers of each cell type in co-cultures, with

168 the phosphatidylinositols $\operatorname{PI}(34: 1), \operatorname{PI}(34: 2)$ found to be the most statistically significant and

169 exhibiting the highest fold change in HeLa cells.

170 In order to assess whether the metabolic profiles can predict the cell type with a single-cell

171 resolution, we evaluated a case of a fibroblast surrounded by HeLa cells (Figure 3D). As shown

172 in Figure 3E, its cell type was predicted correctly. The phosphoethanolamine PE(40:6), the

173 fibroblast marker for NIH3T3 with the highest fold change (Figure 3F) is exhibiting a higher

174 intensity in the fibroblast compared to the surrounding HeLa cells. Interestingly, the visualization

175 of the intensities of the most significant HeLa marker PI(34:1) shows high cell-cell heterogeneity

176 among the HeLa cells (Figure 3G), confirming that it is a combination of several metabolites that

177 enables prediction of the HeLa cell type. 
A

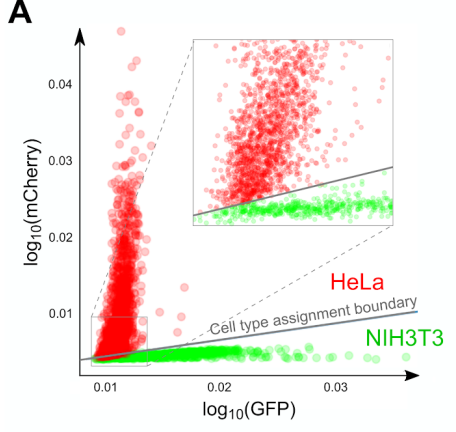

B

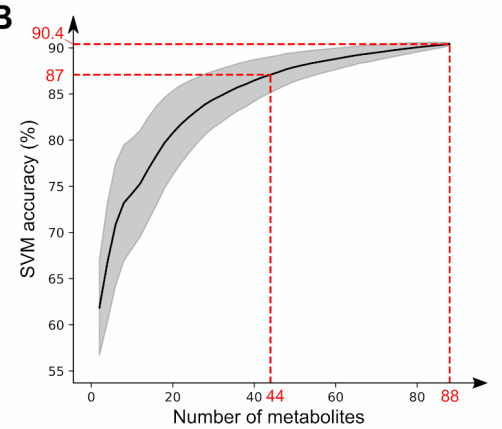

D

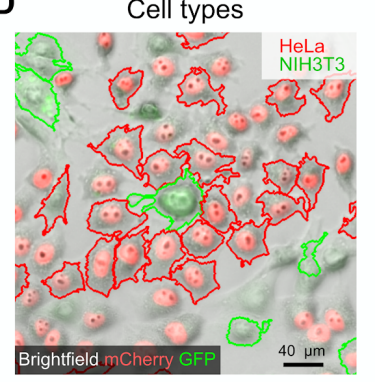

E Predicted cell types

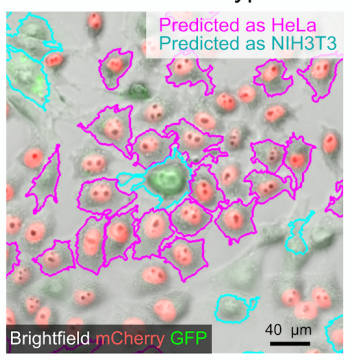

$\mathbf{F}$

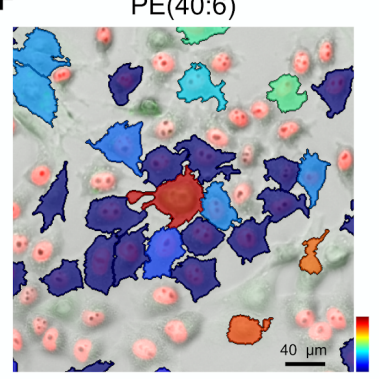

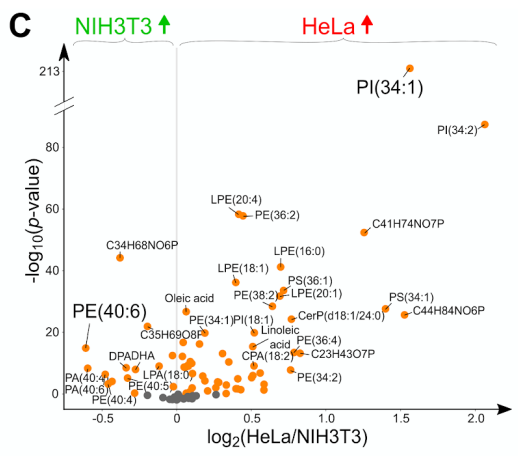

G

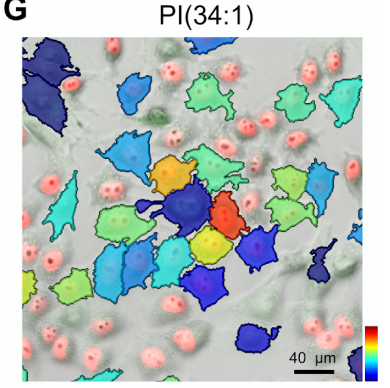

Figure 3. SpaceM can predict the cell type of co-cultured HeLa cells and NIH3T3 fibroblasts

of the cell type for co-cultured HeLa and NIH3T3 cells $(n=3821)$ was done using a linear metabolic profiles, we could predict the cell type with 90.4\% accuracy; even when using only half of the detected metabolites, we could predict the cell type with $87.6 \%$ accuracy on average indicating the richness and the relevance of the detected metabolomes; Support Vector Machine with 10-fold cross validation was used for classification; the plot shows the median accuracy for 1000 random repetitions when subsampling the metabolites, with the grey area showing the confidence intervals of \pm one standard deviation. C: Volcano plot (log2 of the fold change HeLa/NIH3T3 vs. -log10 of the t-test p-value) showing differential properties 88 detected metabolites and lipids. D: Area of the co-cultured cells showing an NIH3T3 cell surrounded by

191 HeLa cells; the sampled cells are outlined according to their assigned type. E: SpaceM 
surrounded by HeLa cells. F-G: Single-cell metabolite images for two markers highlighted in

Figure 2E: phosphoethanolamine PE(40:6) and phosphatidylinositol PI(34:1) validated using the richness of the detected metabolomes and their relevance for each cell type.

\section{Intermixing of metabolomes of co-cultured HeLa and NIH3T3}

Intriguingly, the detected intracellular metabolomes of co-cultured cells were different from

212 we could predict the cell type of co-cultured cells with $90.4 \%$ accuracy, for the monocultured

213 cells we could predict their cell type with a higher accuracy of $96.6 \%$.

214 Furthermore, we discovered that the metabolic intermixing between cells of two types happens

215 locally and can be considered a short-distance effect. The extent of the metabolic intermixing for 
216 NIH3T3 cells depends on the presence of HeLa in their close vicinity (Figure 4D). We estimated

217 that the metabolic intermixing is the strongest at the distance of $58 \mu \mathrm{m}$ for NIH3T3 and $107 \mu \mathrm{m}$

218 for HeLa, with NIH3T3 affected most $\left(r_{s}=0.43,-\log 10(p\right.$-value $)=17.5$ compared to $r_{s}=-0.18$, -

$219 \log 10(p$-value $)=3.8)$. We evaluated whether the observed metabolic intermixing can be observed

220 either due to metabolite delocalization during sample preparation or due to co-ablation of cells.

221 Sample preparation is known to be a key to achieve high spatial resolution MALDI-imaging.

222 Particularly critical is the application of the MALDI matrix, since it can cause metabolite

223 delocalization either during matrix crystallization as crystals can contain analytes from the whole

224 crystal footprint, or due to metabolite leakage while spraying the matrix solution. We have

225 developed a strategy to quantify metabolite delocalization (Figure 4G) that showed that most of

226 metabolites (65 of 88) are well-localized. The delocalized metabolites such as

227 lysophosphoethanolamine LPE(18:0) (see Figure G, cf. well-localized LPE(18:1)) showed only

228 minor levels of delocalization with median distance outside of cells $<5 \mu$ m. Importantly, even

229 when using only well-localized metabolites, SpaceM could predict the cell type with $88.9 \%$

230 accuracy. The fact that the cell type prediction accuracy did not increase after considering

231 localized metabolites only suggests that delocalization does not explain the observed intermixing

232 between the cell types. Next, we evaluated whether co-ablation of cells can be a reason for the

233 observed metabolic intermixing. We considered only the cells which had uniquely-associated

234 ablation marks and excluded 878 cells which were co-sampled (having co-sampling ablation

235 marks, Figure 4H). Still, considering both well-localized metabolites and cells without co-

236 sampling ablation marks only (444 HeLa, 332 NIH3T3) we could predict the cell type with

$23790.7 \%$ accuracy. The lack of the difference in the prediction accuracy (cf. 90.4\% for all

238 metabolites and all cells) suggests that co-sampling of cells does not explain the observed 
241 vicinity and that NIH3T3 cells are stronger affected than HeLa cells.

A

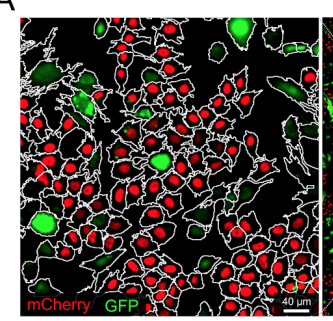

D

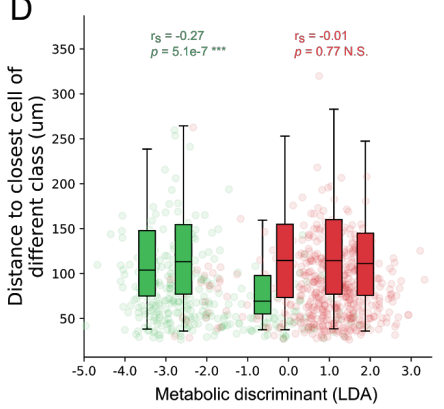

G

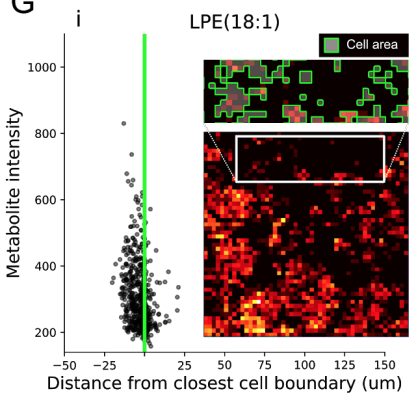

B

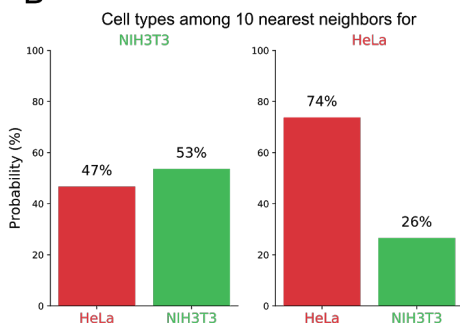

E

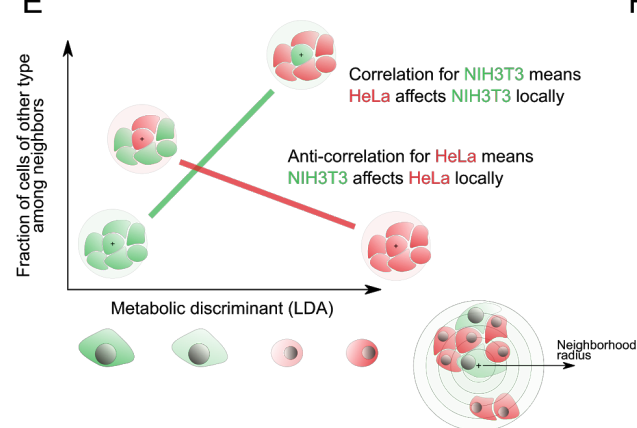

ii

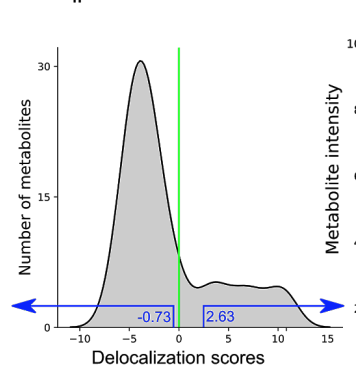

C

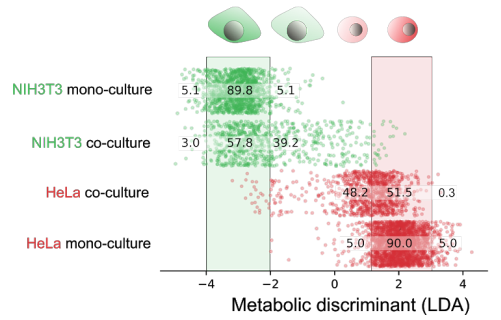

$\mathrm{F}$
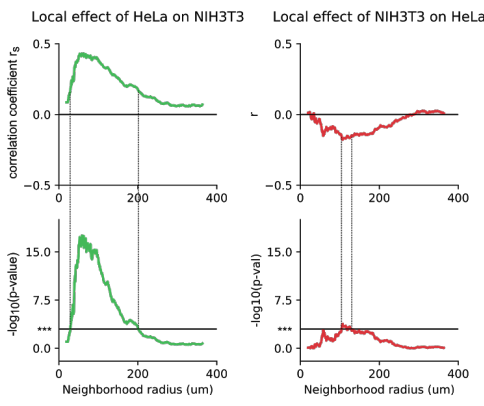

$\mathrm{H}$

Unique ablation mark Co-sampling ablation mark Extracellular ablation mark
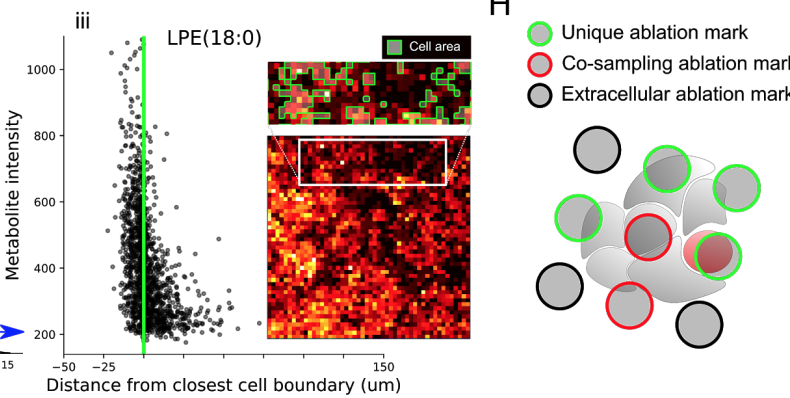

Figure 4. Metabolic intermixing of co-cultured HeLa and NIH3T3 fibroblasts. A: Illustration

244 of the spatial heterogeneity of co-cultured HeLa (H1B-mCherry, red) and NIH3T3 (GFP, green).

245 B: Quantification of the spatial heterogeneity showing that, for each cell type, individual cells are surrounded by cells of other type (on average, an NIH3T3 cell has 47\% HeLa among 10

247 closest neighbors, a HeLa cell has 26\% NIH3T3). C: Less pronounced difference between HeLa and NIH3T3 cells in co-cultures compared to their monocultured counterparts suggests 
249 metabolic intermixing between the cell types, here visualized with the values of the Linear

250 Discriminant Analysis (LDA) discriminant of the single-cell metabolic profiles; the cells are

251 colored and plotted against its cell type and whether they were cultured in co-or mono-culture;

252 the cell type for co-cultured cells was determined using their fluorescence; the vertical bars

253 indicate the 90\%-confidence intervals for each monocultured cell type. D: The metabolomes of

254 NIH3T3 cells are affected most when they have HeLa cells in close vicinity with distance

255 between cell centers of 50-100 $\mu \mathrm{m}$, whereas the average diameter of a HeLa cell equal to 6.7

256 (+/- 1.7) $\mu \mathrm{m}$ and of NIH3T3 equal to $8(+/-2.47) \mu m$. E: Illustration explaining the calculation

257 of the distance of the metabolic intermixing effect, per cell type: For each cell, we consider a

258 neighborhood of particular radius, and calculate a fraction of cells of the other type in this

259 neighborhood. The radius for which this property is most correlated (for NIH3T3 cells) or anti-

260 correlated (for HeLa cells) with the metabolic discriminant (LDA) corresponds to the distance of

261 the intermixing effect. F: NIH3T3 cells are most affected by HeLa at the distance of $58 \mu \mathrm{m}$;

262 HeLa cells are mainly affected by NIH3T3 cells at the distance of $120 \mu \mathrm{m}$, with the correlation

263 coefficient and p-values indicating the smaller extend of this effect compared to NIH3T3. G:

264 Evaluation of the metabolite delocalization confirmed that the metabolic intermixing cannot be

265 explained by the metabolite delocalization between neighboring cells. The scatterplots for

266 ablation marks for lysophosphoethanolamines LPE(18:1) (i) and LPE(18:0) (iii) show how their

267 metabolite intensities depend on the distance to the nearest cell boundary (negative for

268 intracellular ablation marks, positive for extracellular ablation marks). LPE(18:0) exemplifies a

269 delocalized metabolite with the high metabolite intensities observed at the extracellular ablation

270 marks at the distances of $<50 \mu \mathrm{m}$ from cells. LPE(18:1) exemplifies a well-localized metabolite

271 detected predominantly in the intracellular ablation marks only. ii) a histogram of the 
272 delocalization scores for all metabolites showing that most of the metabolites are well-localized;

273 moreover, when considering only well-localized metabolites, SpaceM predicts the cell type with

274 the classification accuracy 88.9\% suggesting that despite a minority of metabolites (23 out of 88)

275 having positive delocalization scores, the metabolic intermixing between the cell types is not

276 explained by the delocalization. $\boldsymbol{H}$ : We confirmed that the metabolic intermixing is not due to the

277 co-sampling of cells (when an ablation mark samples more than one cell e.g. as illustrated with

278 red outlines), since even after considering only well-localized metabolites and excluding co-

279 sampled SpaceM could predict the cell type with $90.7 \%$ accuracy (cf. $90.4 \%$ for all metabolites

280 and cells). *** denotes p-values $<0.001$; N.S. stands for non-significant.

281 SpaceM discovers accumulation of long-chain lipids in steatotic hepatocytes

282 Next, we used SpaceM to investigate the identity of intracellular metabolomes in differentiated

283 human hepatocytes (dHepaRG). During the non-alcoholic fatty liver disease (NAFLD)

284 hepatocytes are known to accumulate lipid droplets (LDs), which in the context of inflammation

285 and necro-inflammation can lead to development of macrovesicular steatosis (Ringelhan et al.

286 2018; Wolf et al. 2014). We could recapitulate macrovesicular steatosis using human or murine

287 hepatocytes in vitro (Wolf et al. 2014). In particular, the pro-inflammatory cytokine $\mathrm{TNF} \alpha$ is

288 known factor promoting steatotic phenotype in hepatocytes (Jung and Choi 2014; Nakagawa et

289 al. 2014). We noticed that the macrovesicular steatosis exhibits a high cell-cell heterogeneity

290 (Figure 5A, Figure S3) and set to characterize the molecular composition of the LDs in steatotic

291 hepatocytes.

292 We cultured monolayers of differentiated human hepatocytes dHepaRG, stimulated them with

293 TNF $\alpha$ in combination with oleic and palmitic acids, and measured LD accumulation using the

294 lipophilic fluorescent dye LD540 (Spandl et al., 2009). After applying SpaceM, we detected 167 
295 metabolites (at an FDR $\leq 10 \%$ ) in 2370 cells. Principal component analysis (PCA) of the single-

296 cell metabolic profiles revealed a significant correlation between the captured metabolome and

297 the LD540 lipid fluorescence (Figure 5B). Triglycerides (TGs), diglycerides (DGs), and

298 phosphatidylcholines (PCs) were identified as the key constituents of the LDs (Figure 5C). This

299 is in line with neutral lipids, mainly DGs and TGs, known to compose the core of hepatic LDs,

300 whereas polar lipids, primarily PCs, to compose the surface (Gluchowski et al., 2017; Ress and

301 Kaser, 2016). Figure 5D shows a single-cell scatterplot for the most correlated lipid TG(50:1)

302 visualizing statistically significant (with $\mathrm{p}$-value $<0.001$ ) correlation of intensities of this lipid

303 with the extent of the macrovesicular steatosis quantified with the LD540 fluorescence.

304 Interestingly, for PCs, sphingomyelins, and phosphoethanolamines, the LD540 fluorescence was

305 found to be positively correlated with the number of carbons suggesting that LDs in steatotic

306 hepatocytes preferentially accumulate long-chain species of these phospholipids (Figure 5E).

307 The opposite effect (negative correlation) was observed for di- and triglycerides albeit not 308 significant (Figure 5F). 
A

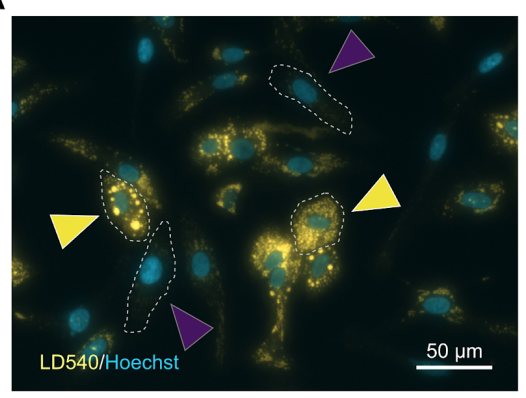

D

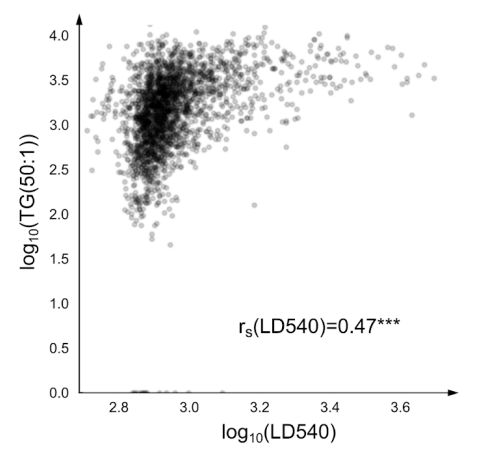

B

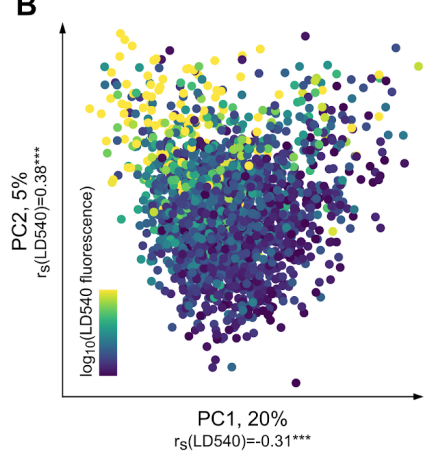

E

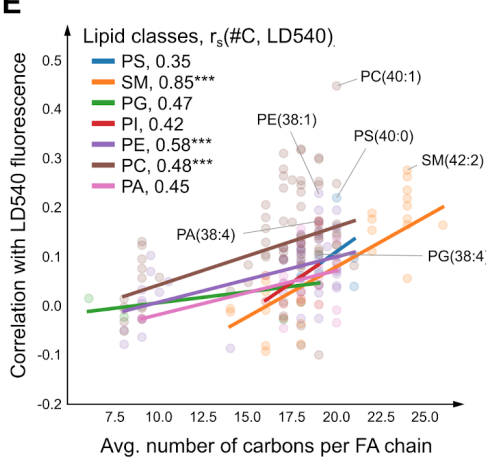

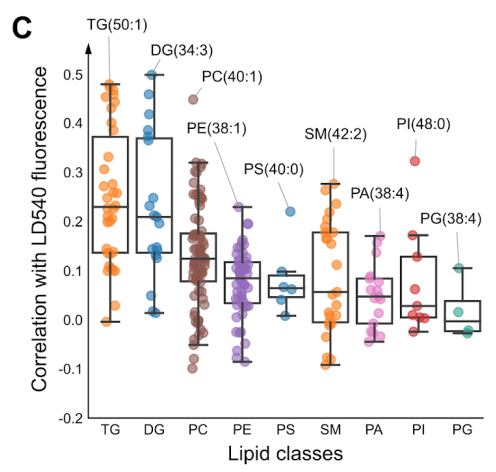

$\mathbf{F}$

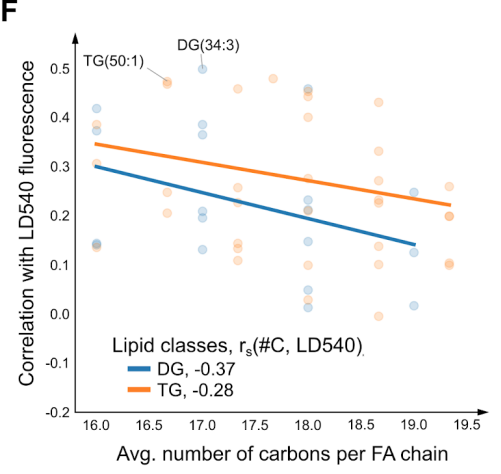

Figure 5. Single-cell analysis of the molecular composition of lipid droplets (LDs) in steatotic steatosis (LDs accumulation) in differentiated human hepatocytes stimulated with TNF $\alpha$ in combination with oleic and palmitic fatty acids; the LD540 lipophilic staining highlights intracellular lipid droplets; Hoechst highlights nuclei; the yellow/blue arrows indicate cells with high/low steatosis. B: Positive Spearman correlations $\left(r_{s}\right)$ the single-cell principal components of the z-scores of the single-cell metabolic profiles between the $\log 10$ of LD540 fluorescence

317 prove that the metabolic profiles represent lipid accumulation. : Accumulation of various lipid

318 classes in LDs as measured by the single-cell Spearman correlations between LD540

319 fluorescence and intensities of 167 detected lipid species; tri- (TG), di-glycerides (DG), and 320 phosphocholines (PC) are the most correlated that is in line with them known to compose the 321 core $(T G, D G)$ and surface (PC) of hepatic LDs. D: Single-cell scatterplot of the most-correlated 322 triglyceride TG(50:1), validated using LC-MS/MS (Data S1). E: For phosphocholines, 
sphingomyelins (SMs), and phosphoethanolamines (PEs), the LD540 fluorescence is significantly and positively correlated with the number of carbons $(\# C)$ suggesting that steatotic hepatocytes accumulate long-chain versions of these phospholipids; the number of carbons is computed as an average per fatty acid chain to be comparable between lipid classes. F: The opposite effect (negative correlation) was observed for di- and triglycerides albeit not significant. *** indicates significance with p-value $\leq 0.001$.

\section{SpaceM discovers intracellular co-regulation of oleic and linoleic acid}

330 We previously found that mice fed a diet enriched with oleic and palmitic fatty acids developed

331 key features of human metabolic syndrome, nonalcoholic steatohepatitis (NASH), and NASH-

332 derived hepatocellular carcinoma (Wolf et al., 2014). To investigate on the single-cell level how

333 the metabolome and lipidome of human hepatocytes is affected by different pro-inflammatory

334 factors, we analyzed hepatocytes cultured under the following conditions: (i) CTRL, untreated

335 cells, (ii) FA, cells stimulated with oleic and palmitic fatty acids (opFAs), (iii) LPS, cells

336 stimulated with a pathogen-associated molecular pattern lipopolysaccharide and opFAs and (iv)

337 TNF $\alpha$, cells stimulated with TNFa and opFAs. SpaceM obtained metabolic profiles of 136

338 metabolites for 22258 cells in total with a batch correction applied to three randomized technical

339 replicates per condition (Table S1, Figure S4). LD540 staining of differentiated, stimulated

340 human hepatocytes revealed lipid accumulation and macrovesicular steatosis (Figs. 5A, S6, S8).

341 PCA of single-cell metabolic profiles indicates the captured differences in the metabolomes of

342 untreated and stimulated cells (Figure 6A). The metabolic shifts between different conditions

343 reflected the expected levels of response to the stimuli as (i) opFAs used for stimulation in the

344 FA condition were also supplemented in the LPS and TNF condition, (ii) TNF $\alpha$ specifically

345 induced the strongest TNF-receptor signaling whereas (iii) LPS only secondarily induces TNF 
346 secretion and TNFR signaling at lower levels (Beutler, 2004). We investigated the contributions

347 of individual metabolites to the principal components and found that, as expected, cells cultured

348 with oleic acid accumulated intracellular oleic acid as compared to the untreated cells (Figure

349 6B). Not all molecules showed elevated accumulation with the increase of the stimuli; see e.g.

350 PIP(38:5) in Figure 4C which exhibits similar intensities between CTRL and FA with a clear

351 increase in the TNF and even more in the LPS condition. Interestingly, opposite to oleic acid,

352 linoleic acid (the second of the opFAs stimuli in the FA, LPS, TNF conditions) exhibits

353 decreased levels in the stimulated cells (Figure 6D). Importantly, there is a clear correlation

354 between the levels of the oleic and linoleic acids across all cells (Figure 6E). A similar effect of

355 the inverse relation between oleic and linoleic acids levels was reported in the livers of mice fed

356 a high-fat diet (da Silva-Santi et al., 2016). However, the bulk analysis could not discern whether

357 the effect occurs in different cell subpopulations or is concerted within the same cells. Our

358 single-cell analysis shows that the effect happens indeed within the same cells thus suggesting

359 intracellular co-regulation of oleic and linoleic acid levels. 
A

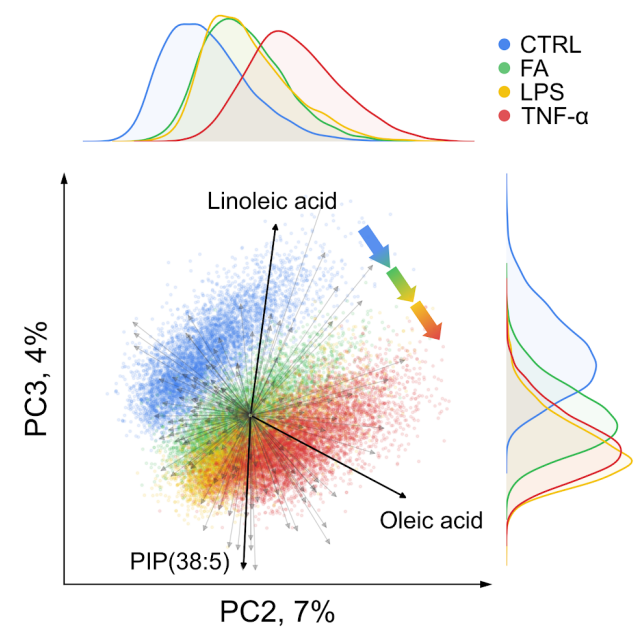

361

\section{Figure 6. Single-cell statistical analysis of steatotic differentiated human hepatocytes}

dHepaRG stimulated with the fatty acids, LPS, and TNFa (n=22258). A: PCA of z-scores of

the single-cell profiles of 136 metabolites; biplot vectors visualize contributions of individual metabolites; gradient-colored arrows illustrate the metabolomes transitions from the untreated cells (CTRL, $n=5654)$ to the cells cultured with oleic and palmitic fatty acids (FA, $n=4972)$, with LPS and the fatty acids (LPS, $n=5062)$, or with TNF $\alpha$ and the fatty acids (TNF $\alpha, n=6570)$. B-D:

Single-cell metabolite intensities mapped onto the PCA plot and the Tukey box plots per condition (25\%-75\% percentiles, whiskers at $1.5 x$ distance of the interquartile range); only cells with non-zero metabolite intensity are shown; see Figure 14 for AMP and glutathione. E: Singlecell scatterplot for intensities of linoleic vs. oleic acids showing an inverse relationship in intracellular levels of these fatty acids upon stimulation and their tight and conditionindependent correlation; the centers of masses and fitted lines are plotted. Oleic acid, linoleic acid, and PIP(38:5) were validated using LC-MS/MS (Data S1).
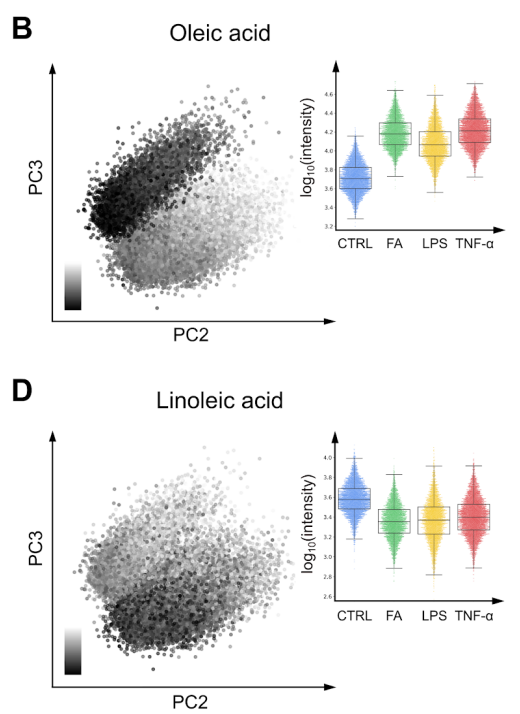

C $\quad \operatorname{PIP}(38: 5)$

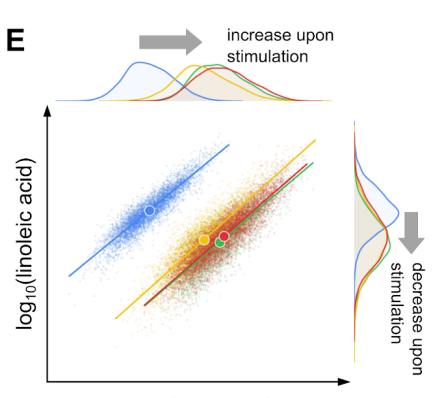

$\log _{10}$ (oleic acid)

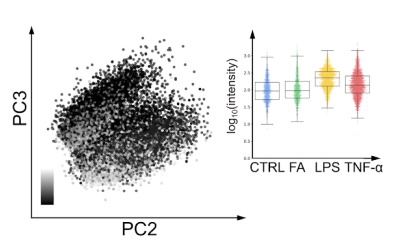




\section{SpaceM discovers association of PIP(38:5) with high cell-cell contact}

378 Finally, we investigated the spatio-molecular organization of human hepatocytes (Figure 7A-D).

379 LD540-fluorescence microscopy revealed lipid accumulation within groups of cells that display

380 high cell-cell contact (Figure S5, S6). Among all the detected metabolites, phosphatidylinositol

381 phosphate PIP(38:4) (although not validated with LC-MS/MS) and PIP(38:5) were most highly

382 associated with cell-cell contact (Figure 7B, Figure S7). PIPs are precursors of PIP3, a signaling

383 phospholipid in the plasma membrane, which might explain their elevation within adjacent cells

384 in a locally-concerted manner. Not all detected metabolites displayed such an association: AMP

385 showed no correlation (Figure 7C) whereas oleic acid showed a negative correlation (Figure 7D).

386 Thus, integrating fluorescent, morphological, spatial, and molecular information can be a

387 powerful approach to explore multi-cellular phenomena (Bray et al., 2016). 
A

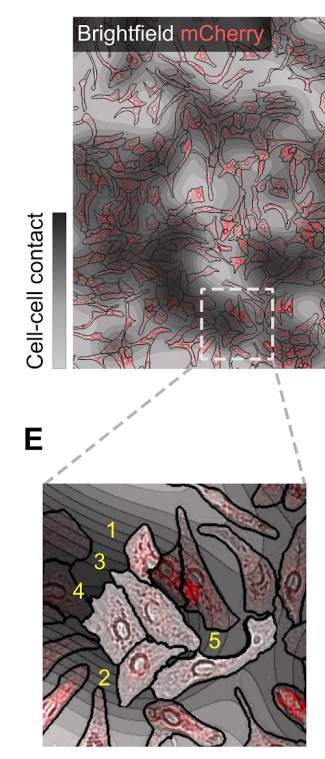

B

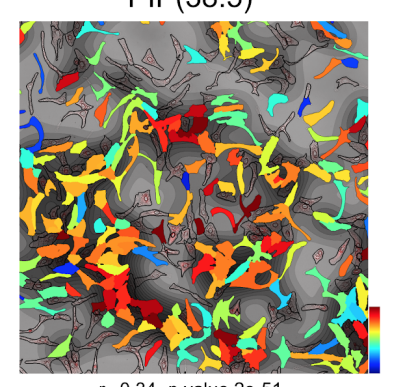

is $0.34, p$-value $2 \mathrm{e}-51$
C

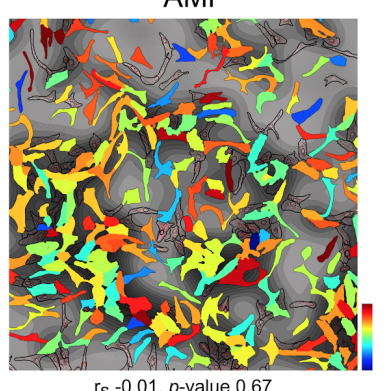

rs $-0.01, p$-value 0.67
D

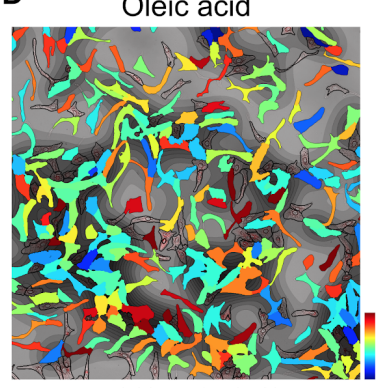

$r_{S}-0.21, p$-value $6 e-20$

F Cell

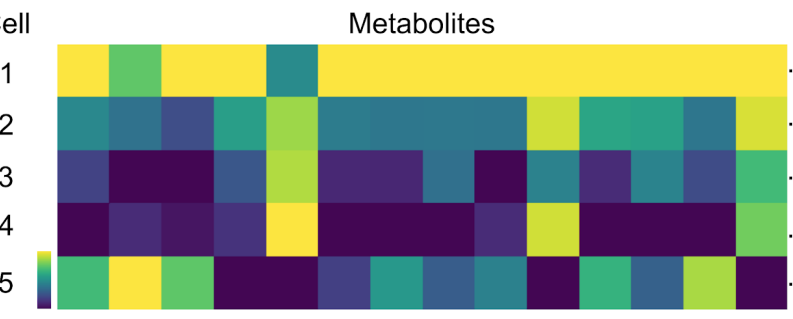

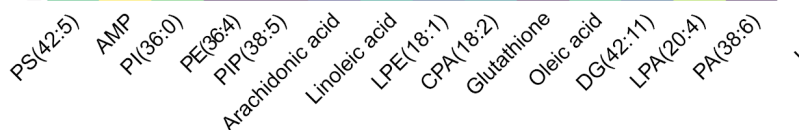

Staining Morpho. Spatial

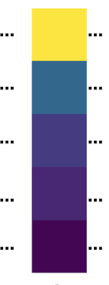

$S^{S^{00}}$

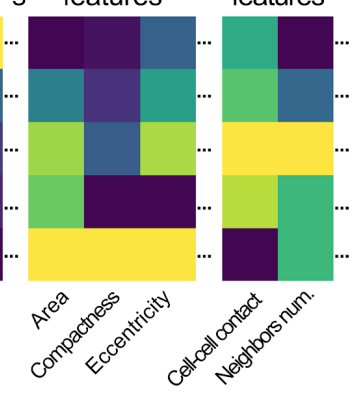

390 Figure 7. Single-cell statistical analysis of steatotic differentiated human hepatocytes

391 stimulated with pro-inflammatory factors (n=22258). A: TNF $\alpha$ hepatocytes with areas of local

392 crowding; the red LD540 fluorescence intensity indicates the accumulation of lipid droplets from

393 low steatosis to macrovesicular steatosis. B-D: Single-cell molecular images for PIP(38:5),

394 AMP, and oleic acid. Next to PIP(38:4), PIP(38:5) is the second most correlated molecule with

395 cell-cell contact, indicating its potential concerted elevation within adjacent cells. Oleic acid,

396 linoleic acid, AMP, glutathione, and PIP(38:5) were validated using LC-MS/MS (Data S1). E-F:

397 Illustration of a part of spatio-molecular matrix for five selected cells that represents single-cell

398 phenotypic information (LD540 fluorescence quantifying the extent of the macrovesicular

399 steatosis), morpho-spatial features (including cell-cell contact), and metabolite intensities. 


\section{Discussion}

401 Here, we presented SpaceM, a novel method for the spatial single-cell analysis of intracellular

402 metabolomes of cultured cells. SpaceM not only detects metabolites in individual cells using

403 MALDI but, critically, integrates MALDI analysis with microscopy and is supplied with data

404 analysis strategies for metabolite annotation, intensities normalization, selection intracellular

405 metabolites and filtering out poor quality cells. The method demonstrated to be robust and

406 reproducible, allowing us to analyze several conditions and replicates, obtaining metabolic

407 profiles of around 100 metabolites from over 30.000 cells.

408 We benchmarked the method by analysing the co-cultured human HeLa cells and mouse

409 NIH3T3 fibroblasts. The single-cell metabolic profiles detected by SpaceM were rich enough to

410 predict the cell type with $90.4 \%$ accuracy with the single-cell resolution. Surprisingly, we

411 detected metabolic intermixing of different cell types upon co-culturing, a yet unreported

412 biological phenomenon. Our investigations indicated that the observed intermixing is a short-

413 distance effect, namely, its extent depends on the presence of cells of other type in close vicinity.

414 We carefully considered all other confounding factors and outruled sampling inaccuracies or

415 metabolite delocalization. All our results indicate that intracellular metabolomes of cells of one

416 type are indeed influenced by the neighboring cells of another type, with NIH3T3 cells affected

417 stronger than HeLa cells.

418 For molecular detection, SpaceM exploits MALDI-imaging thus inheriting the advantages of this

419 technique, in particular the capacity for untargeted metabolomics. We demonstrated it by

420 detecting profiles encompassing 88 (for HeLa and NIH3T3) and 136 (for dHepaRG) metabolite

421 annotations on the level 2 according to the Metabolomics Standards Initiative (Sumner et al., 
422 2007). The use of METASPACE for metabolite annotation was instrumental in the

423 interpretation, quality control, and fast access to the metabolite images of the collected MALDI-

424 imaging data, as METASPACE efficiently reduces millions of mass-to-charge values to tens of

425 metabolite annotations in a False Discovery Rate-controlled manner. SpaceM is not limited to

426 lipids, fatty acids and such small molecules as AMP or glutathione (Figure S8, Data S1) but can

427 be extended to other molecular classes by using another MALDI-imaging protocol. In the HeLa-

428 NIH3T3 co-culture benchmarking experiment, even when using just a half of the 88 detected

429 metabolites, we could predict the cell type with $87.6 \%$ accuracy that affirms the richness of the

430 metabolic profiles detected by SpaceM.

431 SpaceM was enabled by MALDI-imaging achieving the single-cell spatial resolution. However,

432 it is almost impossible to sample highly-confluent cell monolayers without co-ablating several

433 cells at once. Moreover, the sample preparation for MALDI-imaging, particularly matrix

434 application, can negatively affect the spatial resolution. Thus, we set to prove the single-cell

435 nature of the method, especially because it was essential to exclude possible technical reasons for

436 the observed metabolic intermixing between HeLa and NIH3T3. For this, we developed a

437 strategy to consider for each metabolite its delocalization outside the cell perimeter. This led to

438 revelation that, first, the extent of delocalization is metabolite-specific, and second, even for the

439 minority of metabolites characterized as delocalized, the median delocalization is comparable to

440 the average size of a single cell. Moreover, in our benchmarking experiment, discarding

441 delocalized metabolites did not affect the accuracy of the cell type prediction much. Altogether,

442 these novel delocalization analyses confirm the single-cell nature of the SpaceM method. We

443 hypothesize that the delocalization happens during spraying the MALDI matrix solution but do

444 not have yet a definite answer what makes some metabolites delocalized while the majority of 
445 metabolites were detected well-localized within the cell perimeter. For example,

446 lysophosphoethanolamine $\operatorname{LPE}(18: 1)$ was found to be well-localized whereas the structurally

447 similar but saturated LPE(18:0) was found to be delocalized.

448 Compared to other reported single-cell metabolomics methods, e.g. microwells (Ibáñez et al.,

449 2013) or microsampling (Guillaume-Gentil et al., 2017) approaches, SpaceM analyzes cells in

450 situ in their native spatial context, ensures minimal unwanted perturbation, and preserves

451 information about the microenvironment and spatial organization of cells. In contrast to micro-

452 and nano-sampling methods, SpaceM is also a high-throughput method able to analyze over

45310000 cells and at the same time, as we illustrated, detecting rich metabolic profiles. Compared

454 to a microscopy-guided laser ablation approach (Do et al., 2017), SpaceM uses unbiased

455 sampling that facilitates discovery of cell populations which cannot be discriminated by

456 microscopy, helps distinguish intracellular from extracellular signals, and also capitalizes on a

457 softer MALDI ionization better suited for biomolecules. Compared to ultra-high spatial

458 resolution approaches (Passarelli et al., 2017), SpaceM makes possible a high throughput

459 analysis of large populations of cells to investigate their heterogeneity and to discover rare

460 molecular phenotypes. The combination of these strengths makes SpaceM not only a single-cell

461 but also a spatial method. We demonstrated the spatial capacity of SpaceM by discovering short-

462 distance effect of metabolic intermixing between HeLa and NIH3T3 cells and by associating

463 PIPs with high cell-cell contact.

464 We expect SpaceM to be broadly applicable to any adherent cells cultured in a monolayer,

465 avoiding growing on top of each other that can lead to increased co-sampling. In our experience,

466 cell culturing for SpaceM is relatively straightforward and can be evaluated following

467 conventional cell biology practices by paying attention to the cell count, viability and 
468 confluence. SpaceM allows for the determination of cells that are different in their response to

469 changes in the microenvironment, which enables the identification of novel molecular

470 mechanism involved in critical biological processes.

471 SpaceM contributes to the growing field of single-cell -omics methods by providing the missing

472 capacities for spatio-molecular in situ analysis. Future experiments will aim to translate SpaceM

473 to the level of tissue sections. Our method will be particularly useful to investigate health and

474 disease phenomena associated with metabolic reprogramming, spatial organization and/or

475 cellular heterogeneity such as differentiation, infection, drug metabolism, immunity, and cancer.

\section{Acknowledgements}

477 We thank Nassos Typas for advising on biology and providing access to the microscope, Bashir

478 El Debs and Joel Selkrig for training on the microscopy and cell culturing (all EMBL), Andrew

479 Palmer (EMBL) for training and support on MALDI-imaging, Megan Stanifer and Steeve

480 Boulant (DKFZ) for their support and training on the cell culturing, METASPACE software

481 development team (EMBL) for creating and supporting the METASPACE software, Carina

482 Beatrice Vibe (EMBL) for her support and feedback on the manuscript, Angela Andersen (Life

483 Science Editors) for scientific editing, Samantha Seah and Christoph Merten (EMBL) for

484 providing the NIH3T3-GFP cell line, Fabian Merkel and Christian Häring (EMBL) for providing

485 the HeLa Kyoto H2B-mCherry cell line. We further thank other members of the Thesis Advisory

486 Committee of L.R.: Anne-Claude Gavin (EMBL) and Britta Brügger (Heidelberg University).

487 This work was supported by the European Union's Horizon 2020 program under the grant

488 agreements 634402 (T.A.) and 667273 (M.H.), the DKFZ-MOST cooperation program (M.H., 
489 M.S.), Darwin Trust of Edinburgh (S.T.), SFB Transregio grants 179, 209, and 1335 (all M.H.),

490 and the ERC Consolidator grants HepatoMetaboPath (M.H.) and METACELL (T.A.).

\section{Author Contributions}

492 L.R. and T.A. conceived the research. L.R. developed the method. T.A. supervised the study.

493 S.T. performed the co-culture experiment. P.P. performed LC-MS/MS validation. M.S. and M.H.

494 contributed with the hepatocytes model, conceived the treatment design. M.S. cultured and

495 prepared hepatocytes. L.R., M.S., M.H. and T.A. interpreted data. L.R. and T.A. wrote the paper.

496 M.S. and M.H. contributed to the paper writing.

\section{Declaration of Interests}

498 L.R. and T.A. are the inventors on a patent application describing a spatial single-cell

499 metabolomics method. T.A. is a consultant and a member of the scientific advisory board of

500 SCiLS, a Bruker company developing software for MALDI-imaging.

501

502 


\section{References}

Altschuler, S.J., and Wu, L.F. (2010). Cellular heterogeneity: do differences make a difference? Cell 141, 559-563.

Baker, T.C., Han, J., and Borchers, C.H. (2017). Recent advancements in matrix-assisted laser desorption/ionization mass spectrometry imaging. Curr. Opin. Biotechnol. 43, 62-69.

Beutler, B. (2004). Inferences, questions and possibilities in Toll-like receptor signalling. Nature $430,257-263$.

Bray, M.-A., Singh, S., Han, H., Davis, C.T., Borgeson, B., Hartland, C., Kost-Alimova, M., Gustafsdottir, S.M., Gibson, C.C., and Carpenter, A.E. (2016). Cell Painting, a high-content image-based assay for morphological profiling using multiplexed fluorescent dyes. Nat. Protoc. $11,1757-1774$.

Buck, M.D., Sowell, R.T., Kaech, S.M., and Pearce, E.L. (2017). Metabolic Instruction of Immunity. Cell 169, 570-586.

Carpenter, A.E., Jones, T.R., Lamprecht, M.R., Clarke, C., Kang, I.H., Friman, O., Guertin, D.A., Chang, J.H., Lindquist, R.A., Moffat, J., et al. (2006). CellProfiler: image analysis software for identifying and quantifying cell phenotypes. Genome Biol. 7, R100.

Folch, J., Lees, M., and Sloane Stanley, G.H. (1957). A simple method for the isolation and purification of total lipides from animal tissues. J. Biol. Chem. 226, 497-509.

Fortin, J.-P., Cullen, N., Sheline, Y.I., Taylor, W.D., Aselcioglu, I., Cook, P.A., Adams, P., measurements across scanners and sites. Neuroimage 167, 104-120. $343-355$.

531 Gripon, P., Rumin, S., Urban, S., Le Seyec, J., Glaise, D., Cannie, I., Guyomard, C., Lucas, J., 532 Trepo, C., and Guguen-Guillouzo, C. (2002). Infection of a human hepatoma cell line by 533 hepatitis B virus. Proc. Natl. Acad. Sci. U. S. A. 99, 15655-15660.

534 Grün, D., and van Oudenaarden, A. (2015). Design and Analysis of Single-Cell Sequencing 535 Experiments. Cell 163, 799-810.

536 Guillaume-Gentil, O., Rey, T., Kiefer, P., Ibáñez, A.J., Steinhoff, R., Brönnimann, R., Dorwling- 
Carter, L., Zambelli, T., Zenobi, R., and Vorholt, J.A. (2017). Single-Cell Mass Spectrometry of Metabolites Extracted from Live Cells by Fluidic Force Microscopy. Anal. Chem. 89, 50175023.

Ibáñez, A.J., Fagerer, S.R., Schmidt, A.M., Urban, P.L., Jefimovs, K., Geiger, P., Dechant, R., Heinemann, M., and Zenobi, R. (2013). Mass spectrometry-based metabolomics of single yeast cells. Proc. Natl. Acad. Sci. U. S. A. 110, 8790-8794. Genome Biol. 17, 29. and nonalcoholic fatty liver disease. Int. J. Mol. Sci. 15, 6184-6223.

Lee, M.-C.W., Lopez-Diaz, F.J., Khan, S.Y., Tariq, M.A., Dayn, Y., Vaske, C.J., Radenbaugh, A.J., Kim, H.J., Emerson, B.M., and Pourmand, N. (2014). Single-cell analyses of transcriptional heterogeneity during drug tolerance transition in cancer cells by RNA sequencing. Proc. Natl. Acad. Sci. U. S. A. 111, E4726-E4735.

Marioni, J.C., and Arendt, D. (2017). How Single-Cell Genomics Is Changing Evolutionary and Developmental Biology. Annu. Rev. Cell Dev. Biol. 33, 537-553.

Merrill, C.B., Basit, A., Armirotti, A., Jia, Y., Gall, C.M., Lynch, G., and Piomelli, D. (2017). Patch clamp-assisted single neuron lipidomics. Sci. Rep. 7, 5318.

Otsu, N. (1979). A threshold selection method from gray-level histograms. IEEE Trans. Syst.

561 Palmer, A., Trede, D., and Alexandrov, T. (2016). Where imaging mass spectrometry stands: high-resolution imaging mass spectrometry. Nat. Methods 14, 57-60. Marshall, P.S., Arlinghaus, H., Alexander, M.R., et al. (2017). The 3D OrbiSIMS-label-free metabolic imaging with subcellular lateral resolution and high mass-resolving power. Nat. Methods 14, 1175-1183. 
573 Pavlova, N.N., and Thompson, C.B. (2016). The Emerging Hallmarks of Cancer Metabolism.

574 Cell Metab. 23, 27-47.

575 Pelkmans, L. (2012). Cell Biology. Using cell-to-cell variability--a new era in molecular biology.

576 Science 336, 425-426.

577 Preibisch, S., Saalfeld, S., and Tomancak, P. (2009). Globally optimal stitching of tiled 3D

578 microscopic image acquisitions. Bioinformatics 25, 1463-1465.

579 Protsyuk, I., Melnik, A.V., Nothias, L.-F., Rappez, L., Phapale, P., Aksenov, A.A., Bouslimani, 580 A., Ryazanov, S., Dorrestein, P.C., and Alexandrov, T. (2018). 3D molecular cartography using 581 LC-MS facilitated by Optimus and 'ili software. Nat. Protoc. 13, 134-154.

582 Ress, C., and Kaser, S. (2016). Mechanisms of intrahepatic triglyceride accumulation. World J. 583 Gastroenterol. 22, 1664-1673.

584 Rubakhin, S.S., Lanni, E.J., and Sweedler, J.V. (2013). Progress toward single cell 585 metabolomics. Curr. Opin. Biotechnol. 24, 95-104.

586 Russell, A.B., Trapnell, C., and Bloom, J.D. (2018). Extreme heterogeneity of influenza virus 587 infection in single cells. Elife 7.

Sharma, U., and Rando, O.J. (2017). Metabolic Inputs into the Epigenome. Cell Metab. 25, 544558.

Sharon, G., Garg, N., Debelius, J., Knight, R., Dorrestein, P.C., and Mazmanian, S.K. (2014). Specialized metabolites from the microbiome in health and disease. Cell Metab. 20, 719-730.

da Silva-Santi, L.G., Antunes, M.M., Caparroz-Assef, S.M., Carbonera, F., Masi, L.N., Curi, R., Visentainer, J.V., and Bazotte, R.B. (2016). Liver Fatty Acid Composition and Inflammation in Mice Fed with High-Carbohydrate Diet or High-Fat Diet. Nutrients 8 . droplets with a new dye, LD540. Traffic 10,1579-1584.

599 Sumner, L.W., Amberg, A., Barrett, D., Beale, M.H., Beger, R., Daykin, C.A., Fan, T.W.-M., 600 Fiehn, O., Goodacre, R., Griffin, J.L., et al. (2007). Proposed minimum reporting standards for 601 chemical analysis Chemical Analysis Working Group (CAWG) Metabolomics Standards 602 Initiative (MSI). Metabolomics 3, 211-221.

603 Wellen, K.E., and Thompson, C.B. (2012). A two-way street: reciprocal regulation of 604 metabolism and signalling. Nat. Rev. Mol. Cell Biol. 13, 270-276.

605 Wishart, D.S., Knox, C., Guo, A.C., Eisner, R., Young, N., Gautam, B., Hau, D.D., Psychogios, 606 N., Dong, E., Bouatra, S., et al. (2009). HMDB: a knowledgebase for the human metabolome. 
607 Nucleic Acids Res. 37, D603-D610.

608 Wolf, M.J., Adili, A., Piotrowitz, K., Abdullah, Z., Boege, Y., Stemmer, K., Ringelhan, M.,

609 Simonavicius, N., Egger, M., Wohlleber, D., et al. (2014). Metabolic activation of intrahepatic

$610 \mathrm{CD} 8+\mathrm{T}$ cells and NKT cells causes nonalcoholic steatohepatitis and liver cancer via cross-talk

611 with hepatocytes. Cancer Cell 26, 549-564.

612 Zenobi, R. (2013). Single-cell metabolomics: analytical and biological perspectives. Science $613342,1243259$.

614 


\section{Methods}

\section{Co-culturing of HeLa and NIH3T3 cells}

617 HeLa Kyoto H2B-mCherry and NIH3T3-GFP cells were cultured at $37{ }^{\circ} \mathrm{C}$ with $5 \% \mathrm{CO}$, and

618 were maintained in high glucose DMEM (1X Pen/Strep) (Gibco/ThermoFisher Scientific, 619 Bremen, Germany) supplemented with 10\% FBS, $100 \mathrm{U} / \mathrm{ml}$ penicillin, $100 \mu \mathrm{g} / \mathrm{ml}$ streptomycin

620 (Gibco) and $1 \mathrm{mM}$ sodium pyruvate (Gibco). Cells were trypsinized with $0.25 \%$ trypsin-EDTA

621 (Gibco) and split 1:10 twice a week. Two technical replicates for the co-cultures and one

622 replicate for monoculture were used. Trypsinized cells were counted and cells were seeded on 4-

623 well-glass labtek chamber slides (Lab-Tek II, CC2) (ThermoFisher Scientific). In the co-culture

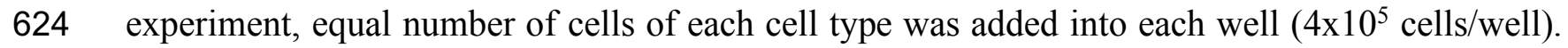

625 After $48 \mathrm{~h}$ of incubation cells were washed with PBS. After washing, the cells were fixed for 15

626 min with 4\% paraformaldehyde (Sigma Aldrich, Darmstadt, Germany) at room temperature.

627 Then the cells were stained with DAPI $(1 \mu \mathrm{g} / \mathrm{ml})$ (ThermoFisher Scientific) in PBS for $20 \mathrm{~min}$ at

628 room temperature.

\section{Hepatocytes culturing and stimulation}

630 HepaRG cell culture and differentiation was performed as described earlier (Gripon et al., 2002).

631 Differentiated HepaRG (dHepaRG) cells were cultured on 4-well-glass chamber slides (Lab-Tek

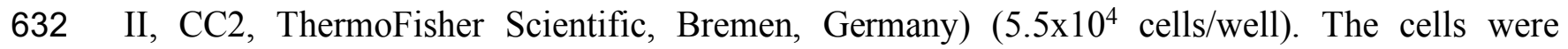

633 stimulated with the fatty acids (opFAs): oleic acid $(66 \mu \mathrm{M})$ and palmitic acid $(33 \mu \mathrm{M})$, opFAs

634 and tumor necrosis-alpha $(\mathrm{TNF} \alpha)$ with the final concentration of $50 \mathrm{ng} / \mathrm{ml}$ (Recombinant Human

635 TNF-alpha, and Systems), or opFAs and lipopolysaccharide (LPS) (100 ng/ml) (LPS from

636 E.coli) (Sigma Aldrich) in Williams E Medium (William's Medium E, with stab. glutamine, 
637 without Phenol Red, with 2,24 g/l NaHCO3) (PAN Biotech) for $24 \mathrm{~h}$. Cells grown in Williams E

638 Medium without supplement for $24 \mathrm{~h}$ were considered as a negative control. For each of those

639 four conditions, cells were seeded in three different wells which were considered as technical

640 replicates (Table S1). After washing, cells were fixed for 15 min with 4\% paraformaldehyde

641 (Sigma Aldrich) at room temperature. Then the cells were washed and stained with Hoechst

$642(1 \mu \mathrm{g} / \mathrm{ml})$ (Hoechst 33342) (ThermoFisher Scientific) and LD540 (0.1 $\mu \mathrm{g} / \mathrm{ml})$ (Spandl et al.,

643 2009) in PBS for $30 \mathrm{~min}$ at room temperature. After washing, cells were stored in $\mathrm{dH} 2 \mathrm{O}$ at $4{ }^{\circ} \mathrm{C}$

644 for one night maximum.

\section{Preparing cells for imaging}

646 The plastic walls of the labtek were removed and the cells were dried in a Lab Companion ${ }^{\mathrm{TM}}$

647 Cabinet Vacuum Desiccator for $16 \mathrm{~h}$ at room temperature and $-0.08 \mathrm{MPa}$. After complete

648 desiccation of the cells, pen marks are manually drawn on the glass slide using a black alcohol

649 pen model 140s black (Edding, Ahrensburg, Germany) to keep track of the glass slide orientation

650 and for image registration. The marks were drawn on the same side as the cells. Cells are kept at

$6514{ }^{\circ} \mathrm{C}$ until analysis. For the following experiments, the samples were analyzed by the microscopy

652 and MALDI-imaging mass spectrometry following a randomized experimental design (Table 653 S1).

\section{Pre-MALDI bright-field and fluorescence microscopy of cells}

657 Fixed cells were sequentially observed with the camera Nikon DS-Qi2 (Nikon Instruments) with 658 the Plan Fluor 10x (NA 0.30) objective (Nikon Instruments) mounted on the Nikon Ti-E inverted 
659 microscope (Nikon Instruments) in bright-field and fluorescence $(620 \mathrm{~nm}$ and $460 \mathrm{~nm})$. The

660 pixel size was $0.73 \mu \mathrm{m}$. The microscope was controlled using the Nikon NIS Elements software.

661 The tiled acquisition of each cell culture area was performed using the JOB functionality of the

662 NIS software. Stitching of tiled frames was performed using the FIJI stitching plugin (Preibisch

663 et al., 2009).

\section{MALDI imaging mass spectrometry}

665 Relative humidity and temperature levels in the mass spectrometry room were monitored and

666 controlled during the whole experiment and were within $44 \%-63 \%$ and $21.1-23.7{ }^{\circ} \mathrm{C}$ (Table S1).

667 For the analysis of the lipid droplets (Figure 3), the 2,5-dihydroxybenzoic acid (DHB) matrix

668 (Sigma Aldrich) $15 \mathrm{mg} / \mathrm{ml}$ dissolved in $70 \%$ acetonitrile was applied onto the dried cells on the

669 labtek slides by using a TM-Sprayer robotic sprayer (HTX Technologies, Carrboro, NC, USA).

670 Spraying parameters were as following: temperature $=100{ }^{\circ} \mathrm{C}$, number of passes $=8$, flow

$671 \mathrm{rate}=0.07 \mathrm{ml} / \mathrm{min}$, velocity $=1350 \mathrm{~mm} / \mathrm{min}$, track spacing $=2 \mathrm{~mm} / \mathrm{min}$, pattern $=\mathrm{CC}$, pressure $=10$

672 psi, gas flow rate $=51 / \mathrm{min}$, drying time $=15 \mathrm{sec}$, nozzle height $=41 \mathrm{~mm}$. The estimated matrix

673 density was of $0.00311 \mathrm{mg} / \mathrm{mm}^{2}$. For investigating the molecular trends within all four

674 considered conditions (Figure 4), the matrix 1,5-diaminonaphthalene (DAN) (Sigma Aldrich)

$67510 \mathrm{mg} / \mathrm{ml}$ dissolved in $70 \%$ acetonitrile was applied onto the dried cells on the labtek slides by

676 using the same TM-Sprayer robotic sprayer. Spraying parameters were as following:

677 temperature $=90 \mathrm{C}^{\circ}$, number of passes $=8$, flow rate $=0.07 \mathrm{ml} / \mathrm{min}$, velocity $=1350 \mathrm{~mm} / \mathrm{min}$, track

678 spacing $=3 \mathrm{~mm} / \mathrm{min}$, pattern $=\mathrm{CC}$, pressure $=10 \mathrm{psi}$, gas flow rate $=2 \mathrm{l} / \mathrm{min}$, drying time $=15 \mathrm{sec}$,

679 nozzle height $=41 \mathrm{~mm}$. The estimated matrix density was of $0.001383 \mathrm{mg} / \mathrm{mm}^{2}$. For MALDI

680 imaging mass spectrometry, the glass slides with the dried cells on them were mounted onto a

681 custom slide adaptor and loaded into the AP-SMALDI source (Transmit, Giessen, Germany). 
682 The MALDI laser focus was optimized manually using the source cameras with the focused

683 beam diameter estimated to be between 15.0 and $43.0 \mu \mathrm{m}$ (mean equal to $29.9 \mu \mathrm{m}$, standard

684 deviation equal to $8 \mu \mathrm{m}$ ). The $\mathrm{x}-\mathrm{y}$ step size (distance between the centers of ablation marks) was

685 set to $50 \mu \mathrm{M}$. For each pixel, the spectrum was accumulated from 30 laser shots at $60 \mathrm{~Hz}$.

686 Negative mode MS analysis was performed in the full scan mode in the mass range of 200-1100

$687 \mathrm{~m} / \mathrm{z}$ (resolving power $\mathrm{R}=140000$ at $\mathrm{m} / \mathrm{z}$ 200) using an QExactive Plus mass spectrometer

688 (ThermoFisher Scientific). MS parameters in the Tune software (version 2.5 Build 2042,

689 ThermoFisher Scientific) were set to the spray voltage of $4.10 \mathrm{kV}$, S-Lens $80 \mathrm{eV}$, capillary

690 temperature $250 \mathrm{C}$. The data was converted from the RAW format into the imzML format

691 containing only centroided data using the ImageQuest software, v.1.1.0 (ThermoFisher

692 Scientific). Metabolite annotation was performed using the METASPACE cloud software

693 (http://metaspace2020.eu) implementing the bioinformatics methods for False Discovery Rate-

694 controlled annotation published by us earlier (Palmer et al., 2017) with the $\mathrm{m} / \mathrm{z}$ tolerance of 3

695 ppm and FDR of 10\%, 20\%, and 50\% against the HMDB metabolite database v2.5 (Wishart et

696 al., 2009).

697 Post-MALDI microscopy to detect MALDI ablation marks

698 The cells were imaged in bright-field microscopy after MALDI-imaging using the same

699 microscopy setup and parameters as described earlier in the pre-MALDI microscopy section to

700 define the positions of the ablation marks with respect to the fiducial marks.

$701 \quad$ Association of laser ablation marks with single cells

702 This is the key part of the method as it solves the challenge that single cells are not visible in the

703 post-MALDI microscopy images due to the opaque layer of MALDI matrix covering cells. Here,

704 ablation marks left by the MALDI laser were associated with single cells in three steps: a) cells 
segmentation in the pre-MALDI microscopy images, b) detection of laser ablation marks in post-

MALDI microscopy images, c) matching between ablation marks and MALDI mass spectra and with the segmented single cells. et al., 2006) where the DAPI staining channel was used to generate seeds for a region growing

711 algorithm detecting cells boundaries in the LD540-staining channel. In step b), we first denoised

712 the bright-field microscopy images by applying a low-pass filter in the 2D Fourier frequency

713 domain, in particular to exploit both the regular distances between ablation marks as well as the

714 repeated shape of the ablation mark itself. Then, we applied a contrast-enhancing filter (using the

715 clip function from the Python module numpy) and Otsu's thresholding method (Otsu, 1979) to

716 binarize the image (using the imbinarize function in Matlab). Then, we applied morphological

717 image analysis operations of closing and then opening to fill in the holes in the image and to 718 remove individual noisy pixels (using the imclose and imopen functions in Matlab). This

719 provided estimations of the centers of mass of each ablation mark (Figure S9). In step c), we fit a

720 theoretical rectangular grid to the ablation marks. The numbers of $\mathrm{X}$ - and $\mathrm{Y}$ - grid steps were

721 defined as set up during the MALDI acquisition. The center of the acquisition region was

722 considered as the center of the grid. The orientation of the grid with respect to the post-MALDI

723 microscopy image was optimized by finding an angle which resulted in the best overlap between

724 the grid lines and the detected ablation marks. The X-and Y-spacing of the grid were optimized

725 by minimizing the distance between the grid nodes and the center of mass of the nearest neighbor

726 ablation mark. Then, only ablation marks which were the nearest neighbors to the grid nodes

727 were taken and re-indexed (Figure S10). This provided X-and Y-coordinates for each ablation 
728 mark associated with a collected MALDI spectrum. In order to improve estimations of the

729 ablations marks areas used later for normalization, their segmentation was further improved by

730 applying a custom region-growing algorithm implemented in Python. In step d), co-registration

731 of pre- and post-MALDI microscopy images was done based on the pen marks drawn on the

732 edge of the wells used as fiducials. We first segmented the pen marks in both pre- and post-

733 MALDI bright-field microscopy images using Otsu's intensity thresholding method. Then, we

734 used the basin-hopping optimization algorithm (Python implementation from the scipy package

$735 \mathrm{v} 0.18 .1)$ to find the best linear transformation matching the coordinates of the edges of the pen

736 marks between the pre- and post-MALDI images (Figure S10). The optimal linear

737 transformation was applied to the post-MALDI microscopy images to map the ablation marks to

738 the pre-MALDI microscopy images. The initial assessment of the co-registration quality and

739 overlaying of the metabolite intensities was performed in the 'ili web app at http://ili.embl.de

740 (Protsyuk et al., 2018).

\section{Single-cell intensity normalization}

742 A normalized intensity of each metabolite in a single cell was constructed as follows. For each

743 cell, we considered all ablation marks overlapping with the cell area and selected the associated

744 ablation marks which overlap with the cell by over than $30 \%$ of their ablation area. The

745 metabolite intensities coming from an ablation mark were normalized by dividing them by the

746 ratio of the sampling area (defined as the number of pixels of the intersection of the ablation

747 mark and any cell region) to the area of the ablation mark. Finally, for each cell its normalized

748 metabolite intensities were calculated as the weighted average normalized intensities of the

749 associated ablation marks where the weights are defined as the ratio of the shared pixels (Figure

750 2). In order to account for the variations in permeabilization efficiency between the biological 
751 replicates, single-cell LD540 fluorescence intensities were normalized by dividing them by the

752 median DAPI intensity (median over a well).

\section{Selecting intracellular metabolites}

754 We selected metabolite annotations corresponding to intracellular metabolites as follows. First,

755 for each ablation mark we assigned to it the inside-cells label having values either of zero or one

756 based on whether the mark has any overlap with any cell. Then, for each metabolite ion image,

757 its intensities were binarized to zero-one values by selecting a threshold leading to the highest

758 Pearson correlation with the inside-cells labels. The threshold value was found using the basin-

759 hopping optimization algorithm. In order to consider only intracellular metabolites for further

760 analysis, we selected those metabolite annotations whose binarized ion images were correlated

761 with the inside-cells labels with the Pearson correlation higher than 0.25 . For the stimulated

762 dHepaRG experiment where three replicates for each of four conditions were obtained, we

763 considered the metabolite annotations which were shared by at least three samples (out of 12

764 overall) that led to 136 annotations. For each metabolite annotation, we pulled the ion images

765 with the $\mathrm{m} / \mathrm{z}$ tolerance of $3 \mathrm{ppm}$ from the imzML files.

\section{Cell filtering and batch correction}

767 We filtered out 5\% of cells with the lowest metabolite yield, namely the cells which had most

768 zero-valued metabolites annotations, following the approach well-accepted in single-cell

769 transcriptomics (Grün and van Oudenaarden, 2015) (Figure S11). In the stimulated HepaRG

770 experiment, this filtered out 1240 cells out of 23498 overall. In the stimulated HepaRG

771 experiment, to compensate for the batch effect between the biological replicates within each

772 condition, we applied the combat batch correction algorithm (Fortin et al., 2017) originally 
773 developed for single-cell transcriptomics data using its open-source Python implementation

774 neuroCombat available at https://github.com/ncullen93/neuroCombat (Figure S4).

775 Cell type classification for the co-culture experiment

776 The assignment of the cell type based on the constitutive fluorescence of the cells (mCherry for

777 HeLa, GFP for NIH3T3) was done by finding a separating linear boundary between the two

778 populations (Figure 2A). The resulting cell types provided the ground truth labels for the Linear

779 Discriminant Analysis (LDA) performed using the Python scikit-learn LDA implementation

780 (version 0.19.1).

781 LC-MS/MS validation of METASPACE annotations

782 Sample preparation: Lipids and fatty acids were extracted using the Folch method (Folch et al.,

783 1957) with chloroform:methanol (2.5:1). For lipidomics analysis, the dried samples were

784 reconstituted in isopropanol:methanol (1:1) and injected $10 \mathrm{uL}$ into the LC-MS system. For

785 metabolomics analysis, metabolites were extracted in $80 \%$ methanol and directly injected $20 \mathrm{uL}$

786 into the LC-MS system.

787 LC-MS/MS methods for lipidomics: All LC-MS/MS analyses were performed on a Vanquish

788 Ultra-High Performance Liquid Chromatography (UHPLC) system coupled to a Q-Exactive Plus

789 High Resolution Mass Spectrometry (HRMS) (ThermoFisher Scientific) with an electrospray

790 ionization (ESI) source operated in either positive or negative mode. The separation of lipids and

791 fatty acids was carried out using an Agilent Poroshell EC-C 18 column ( 3 x $50 \mathrm{~mm} ; 2.7 \mu \mathrm{M})$

792 maintained at $40{ }^{\circ} \mathrm{C}$ at the flow rate of $0.26 \mathrm{ml} / \mathrm{min}$. The mobile phase consisted of solvent A

793 (acetonitrile-water (4:6)) and solvent B (isopropyl alcohol-acetonitrile (9:1)), which were

794 buffered with either $10 \mathrm{mM}$ ammonium acetate (for negative mode) or $10 \mathrm{mM}$ ammonium 
795 formate acidified with $0.1 \%$ formic acid (for positive mode). The UHPLC gradient was set at $79620 \%, 20 \%, 45 \%, 52 \%, 66 \%, 70 \%, 75 \%, 97 \%, 97 \%, 20 \%, 20 \%$ of solvent $\mathrm{B}$ at the time points 0 ,

$7971.5,4,5,7,8,10,12,15,16,19$ min, respectively. Fatty acids and lipids were detected with the

798 HRMS full scan at the mass resolving power $\mathrm{R}=35000$ in the mass range of $100-1500 \mathrm{~m} / \mathrm{z}$. The

799 data-dependent tandem (MS/MS) mass scans for five most intense ions (TOP5) were obtained

800 along with full scans using higher energy collisional dissociation (HCD) with normalized

801 collision energies of 20,30 and 40 units at the mass resolving power $\mathrm{R}=17500$. The MS

802 parameters in the Tune software (ThermoFisher Scientific) were set as: spray voltage of $4 \mathrm{kV}$,

803 sheath gas 30 and auxiliary gas 5 units, S-Lens $65 \mathrm{eV}$, capillary temperature $320^{\circ} \mathrm{C}$ and

804 vaporization temperature of auxiliary gas was $300^{\circ} \mathrm{C}$.

805 LC-MS/MS methods for metabolomics: LC-MS/MS metabolomics analysis was carried out

806 using an Xbridge BEH Amide column $(100 \mathrm{X} 2.1 \mathrm{~mm} ; 2.5 \mu \mathrm{M})$ maintained at $40{ }^{\circ} \mathrm{C}$ at the flow

807 rate of $0.3 \mathrm{ml} / \mathrm{min}$. The mobile phase consisted of solvent $\mathrm{A}(7.5 \mathrm{mM}$ ammonium acetate with

$8080.05 \% \mathrm{NH} 4 \mathrm{OH}$ ) and solvent B (acetonitrile). The UHPLC gradient was set at 85\%, 85\%, 10\%,

$80910 \%, 85 \%, 85 \%$ of solvent $\mathrm{B}$ at the time points $0,2,12,14,14.1,6$ min, respectively.

810 Metabolites were detected with HRMS full scan at the mass resolving power $\mathrm{R}=70000$ in mass

811 range of 60-900 m/z. The data-dependent MS/MS mass scans were obtained along with full scans

812 using HCD of normalized collision energies of 10, 20 and 30 units which were at the mass

813 resolving power $\mathrm{R}=17500$. The MS parameters in the Tune software (ThermoFisher Scientific)

814 were set as: spray voltage of $4 \mathrm{kV}$ (for negative mode $3.5 \mathrm{kV}$ ), sheath gas 30 and auxiliary gas 5

815 units, S-Lens $65 \mathrm{eV}$, capillary temperature $320^{\circ} \mathrm{C}$ and vaporization temperature of auxiliary gas

816 was $300{ }^{\circ} \mathrm{C}$. Data was acquired in the full scan mode and MS/MS mass spectra for TOP5

817 precursor ions. 
818 LC-MS/MS validation of METASPACE annotations: LC-MS/MS validation of lipid and

819 metabolite METASPACE annotations was performed either by comparing retention times, exact

$820 \mathrm{~m} / \mathrm{z}$ (MS) and fragmentation pattern (MS/MS) spectra with authentic standards or by matching

821 MS/MS spectra with the EMBL Metabolomics Core Facility (MCF) spectral library (available at

822 http://curatr.mcf.embl.de/) and public spectral libraries (LipidBlast, LIPID MAPS and mzCloud).

823 The details of annotation validation are summarized in Supplementary Data S1. The structural

824 annotation procedure for head groups (HD) and fatty acid side chains (SD) is described in details

825 in (Palmer et al., 2017).

\section{Data visualization}

827 All plots were generated in Python, version 3.6.2, by using the packages matplotlib 2.1 and 828 seaborn 0.8.1. The Python package scikit-learn 0.19.1 was used for the Principal Component 829 Analysis.

\section{Data availability}

831 All metabolite and lipid annotations and images are publicly available at the METASPACE

832 online knowledgebase (URL for the co-cultured and mono-cultured HeLa and NIH3T3 cells

833 from Figure 2, URL for the dHepaRG cells from Figure 3, $\underline{\mathrm{URL}}$ for the dHepaRG cells from

834 Figure 4).

835 


\section{Supplementary Figures}

1 Cell preparation

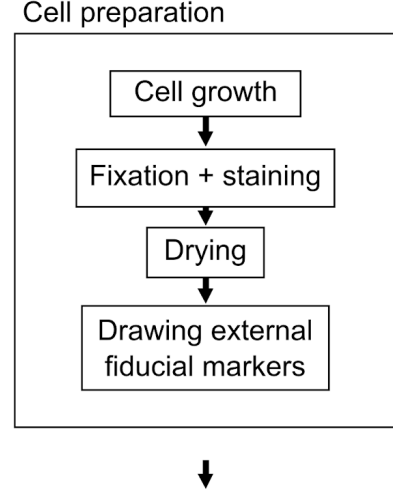

2

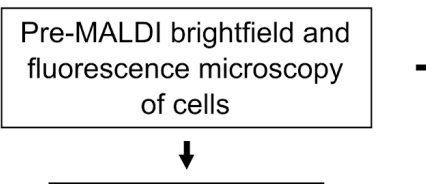

3

MALDI imaging mass spectrometry

$\downarrow$

4

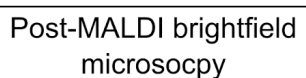
microsocpy

5 Association of laser ablation marks with single-cells

a) Single-cell segmentation $+\quad$\begin{tabular}{c}
$\begin{array}{c}\text { Fiducial markers } \\
\text { segmentation }\end{array}$ \\
\hline
\end{tabular}

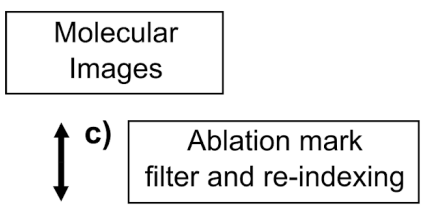

b)

\begin{tabular}{|c|c|}
\hline $\begin{array}{c}\text { Ablation mark } \\
\text { segmentation }\end{array} \quad+\quad \begin{array}{c}\text { Fiducial markers } \\
\text { segmentation }\end{array}$ \\
\hline
\end{tabular}

842 Supplementary Figure S1. Detailed workflow of the SpaceM method, see Figure 1 for a 843 visualization of the workflow supplemented with visual elements. 


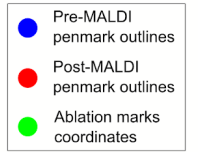

Stitched microscopy images

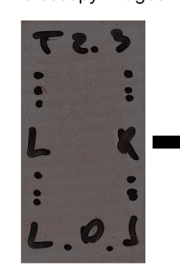

西 Overlay of penmarks outlines

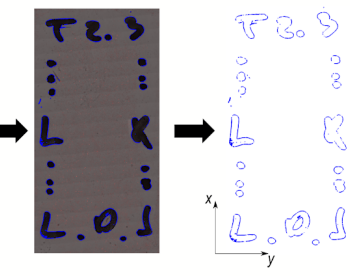

Post-MALDI microscopy
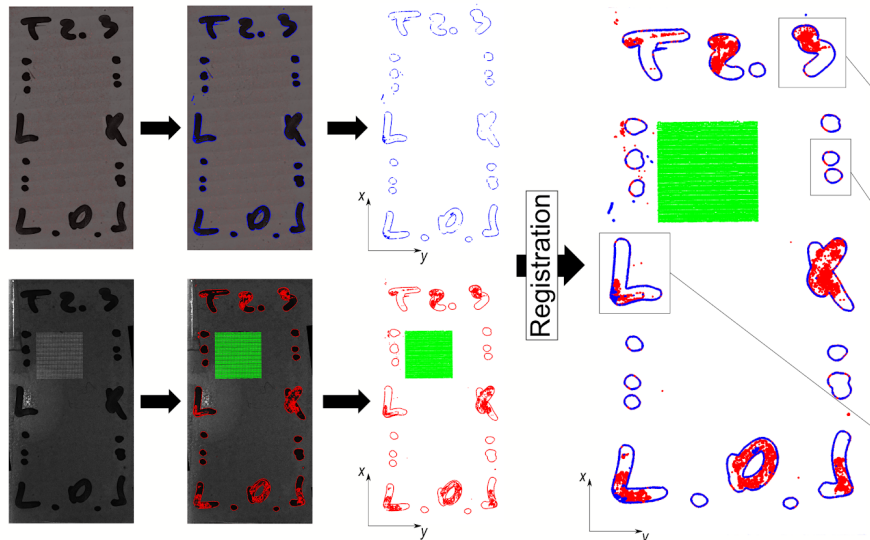

Ablation marks coordinates overlaid onto pre-MALDI microscopy image
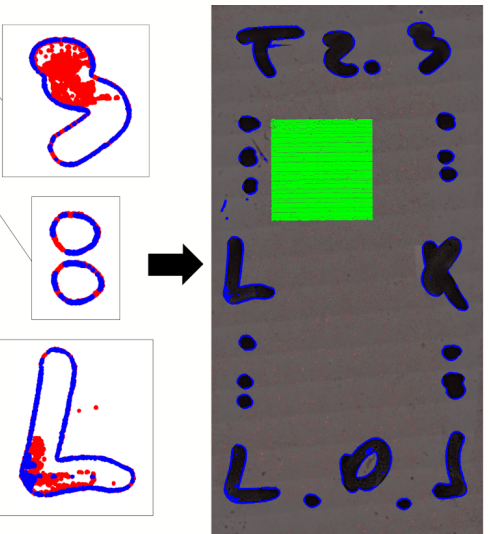

Supplementary Figure S2. Registration workflow of pre- and post-MALDI microscopy images.

847 The area where MALDI-imaging was applied is shown in green. The features of the pen marks 848 used as fiducials are shown in blue (for pre-MALDI microscopy images) or red (for post849 MALDI microscopy images).
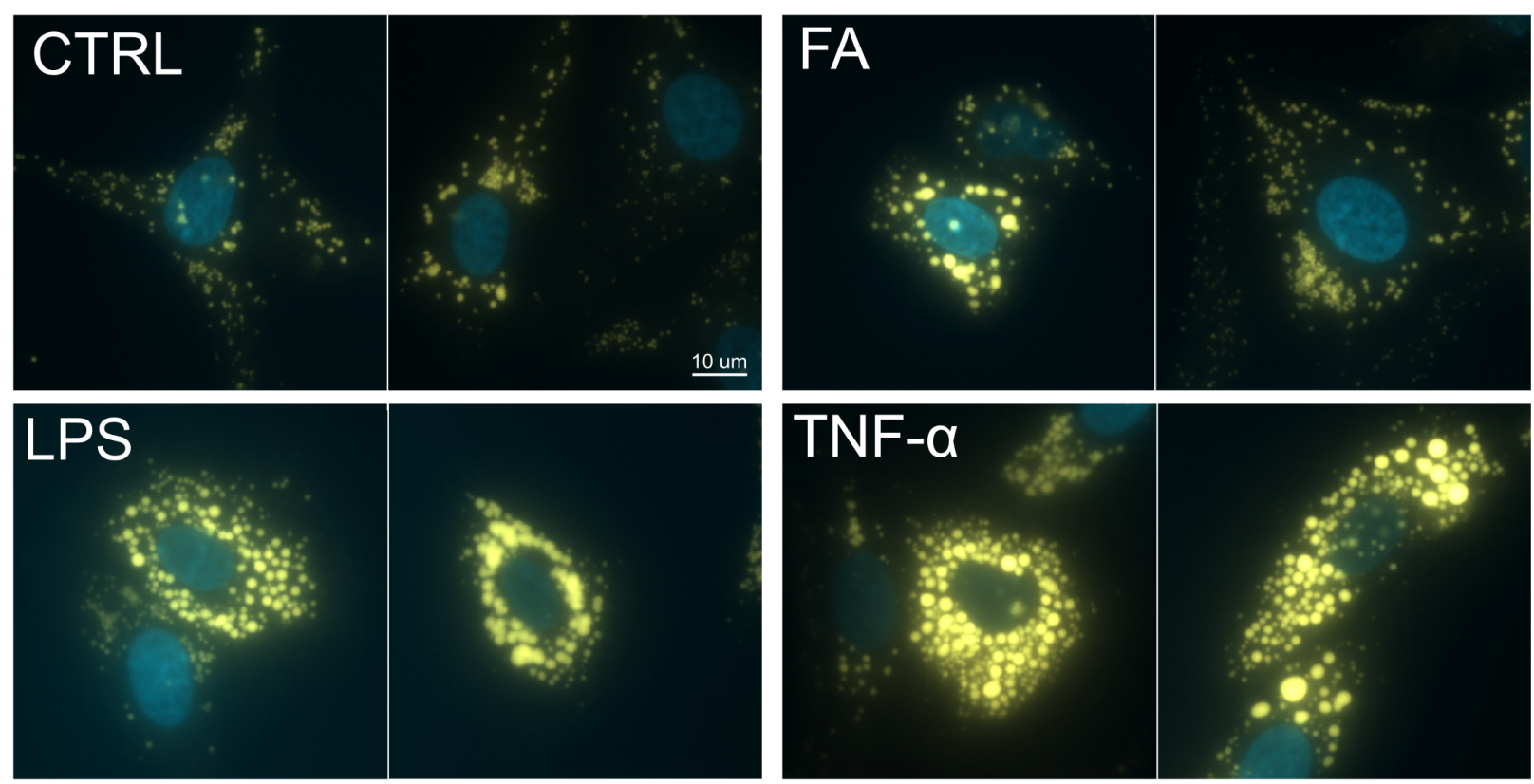

852 Supplementary Figure S3. Lipid accumulation, also known as macro-vesicular steatosis, in

$853 \mathrm{dHepaRG}$ hepatocytes both inherently as well as under stimulation with: oleic and palmitic fatty 
854 acids (FA), LPS in combination with the fatty acids, TNFa in combination with the fatty acids

855 (yellow for LD540, blue for DAPI) to highlight the localization around nucleus. Each subplot

856 shows two illustrative examples of cells.

857

A

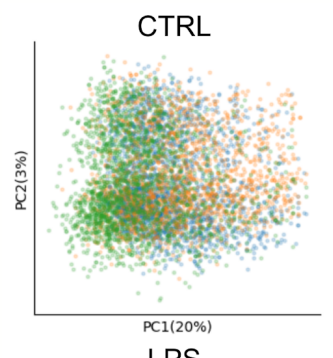

LPS

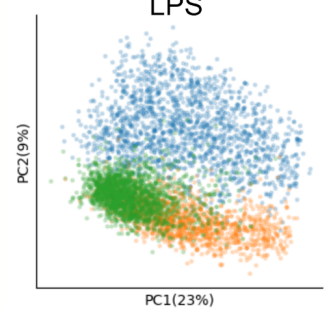

Before batch correction
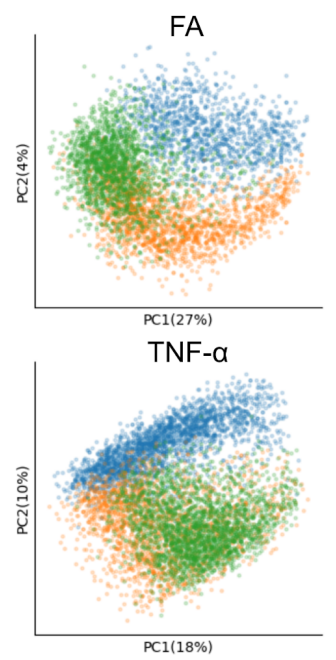

B
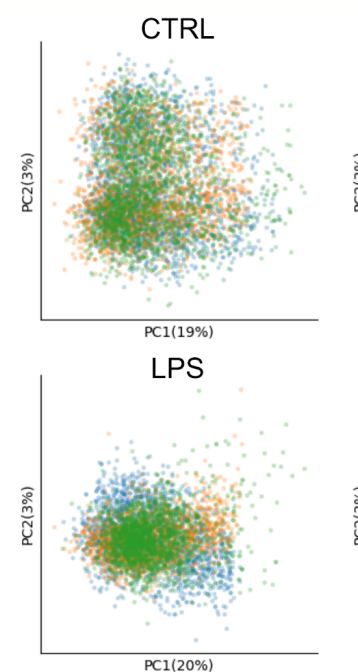

FA

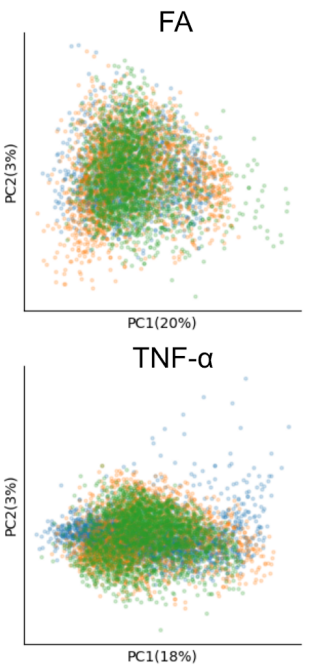

859 Supplementary Figure S4. Batch correction of variability between technical replicates in the

860 stimulated dHepaRG experiment by using the combat algorithm. The plots show a PCA plot of

861 the single-cell metabolic profiles with cells color-coded according to the replicate. A: Before

862 batch correction, B: after batch correction. 


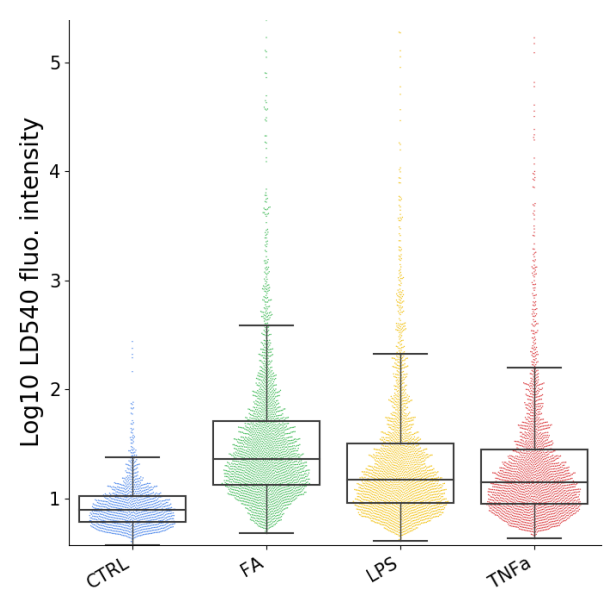

865 Supplementary Figure S5. Swarming plots of single-cell LD540-fluorescent intensities of 866 stimulated dHepaRG cells for each condition indicating increased lipid accumulation upon 867 stimulation with FAs, LPS, and TNF $\alpha$.

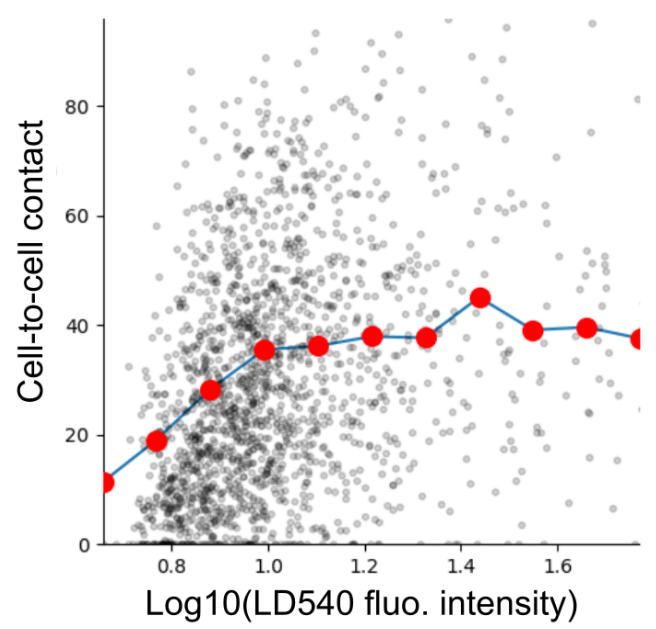

870 Supplementary Figure S6. Scatter plot of the LD540 fluorescent intensity and cell-cell contact

871 for one replicate of $\mathrm{dHepaRG}$ cells stimulated with $\mathrm{TNF} \alpha$ in combination with oleic and linoleic

872 acid $(n=1830)$, with the Spearman $r_{s}$ 0.34, p-value 9.18e-4. The LD540 intensities were

873 thresholded at the $95 \%$ percentile. Red dots visualize average values for regularly-spaced bins of

874 LD540 fluorescence intensity. 

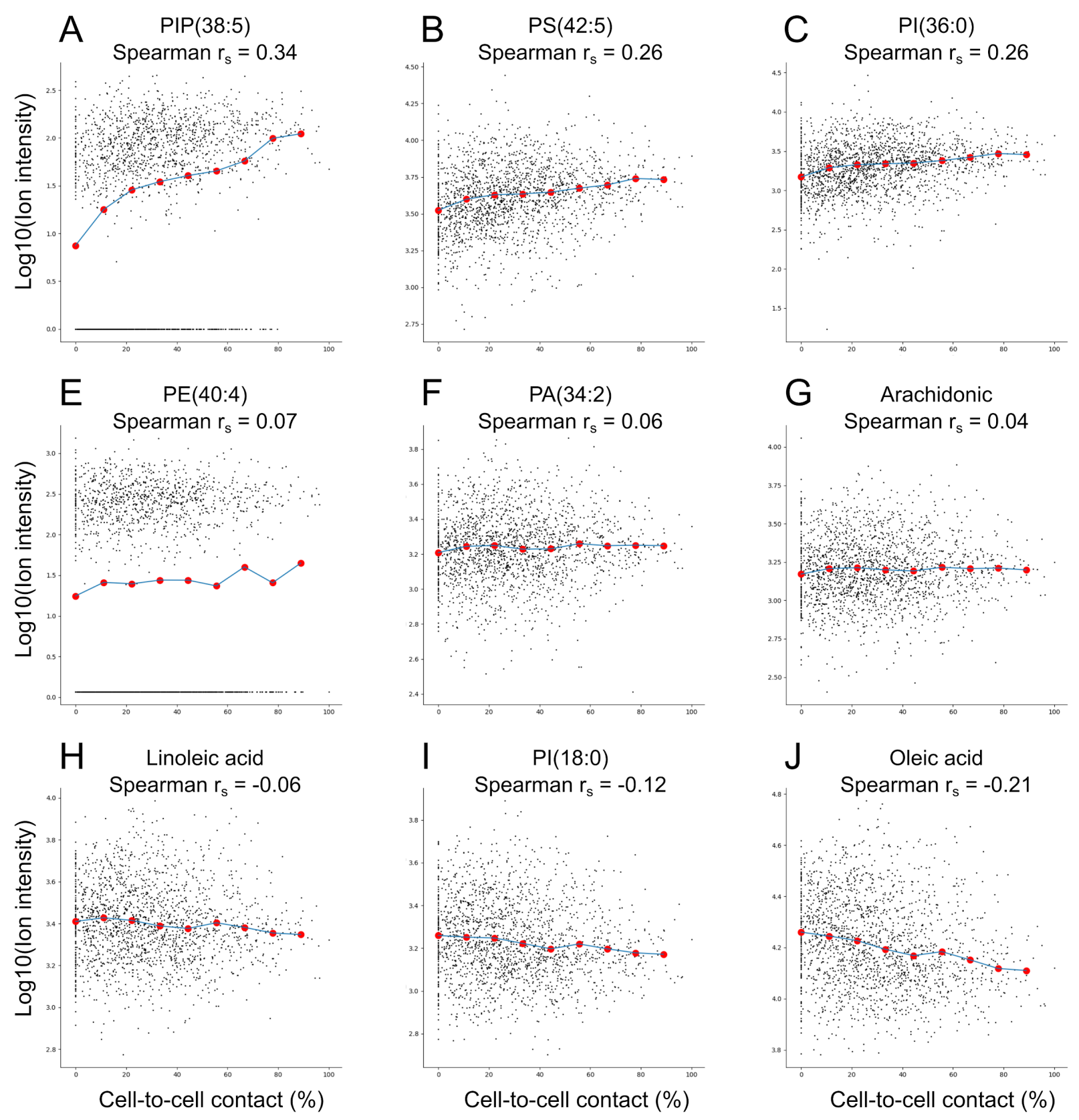

876 Supplementary Figure S7. Observed relationship between local crowding and intracellular

877 metabolite intensity. One dot represents one cell. Red dots represent average intensities for

878 regular bins of local crowdedness. The data are coming from one replicate of the TNF $\alpha$ condition

879 (1830 cells). 

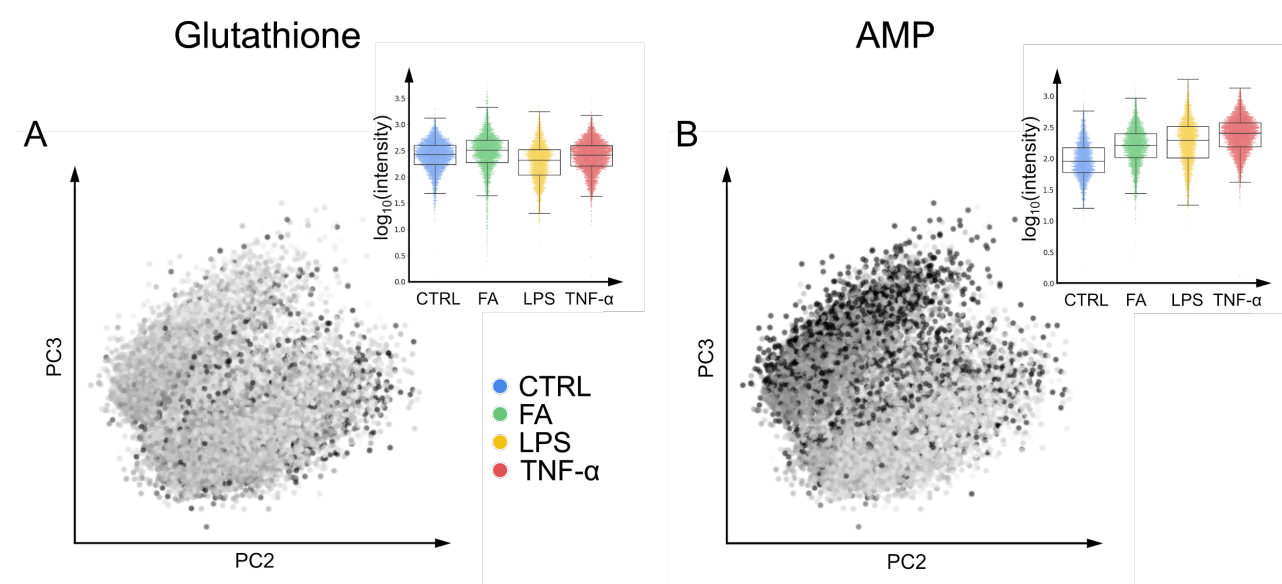

882 Supplementary Figure S8. Mapping of intensities of the small molecules glutathione and AMP

883 onto the principal components of z-scores of the single-cell metabolic profiles of dHepaRG cells

884 from the CTRL, FA, LPS, and TNF $\alpha$ conditions (similar to Figure 3B-D).

885

886
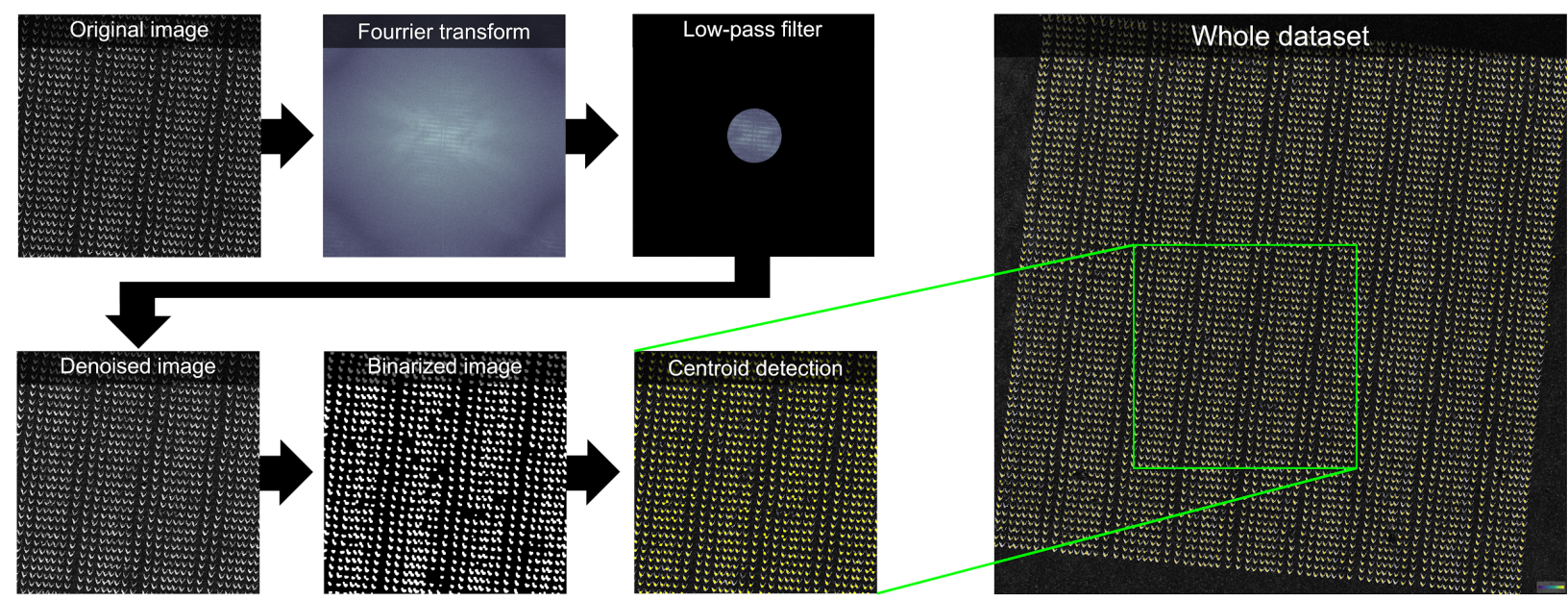

Supplementary Figure S9. Illustration of the procedure for detection of laser ablation marks in

889 post-MALDI microscopy images. 

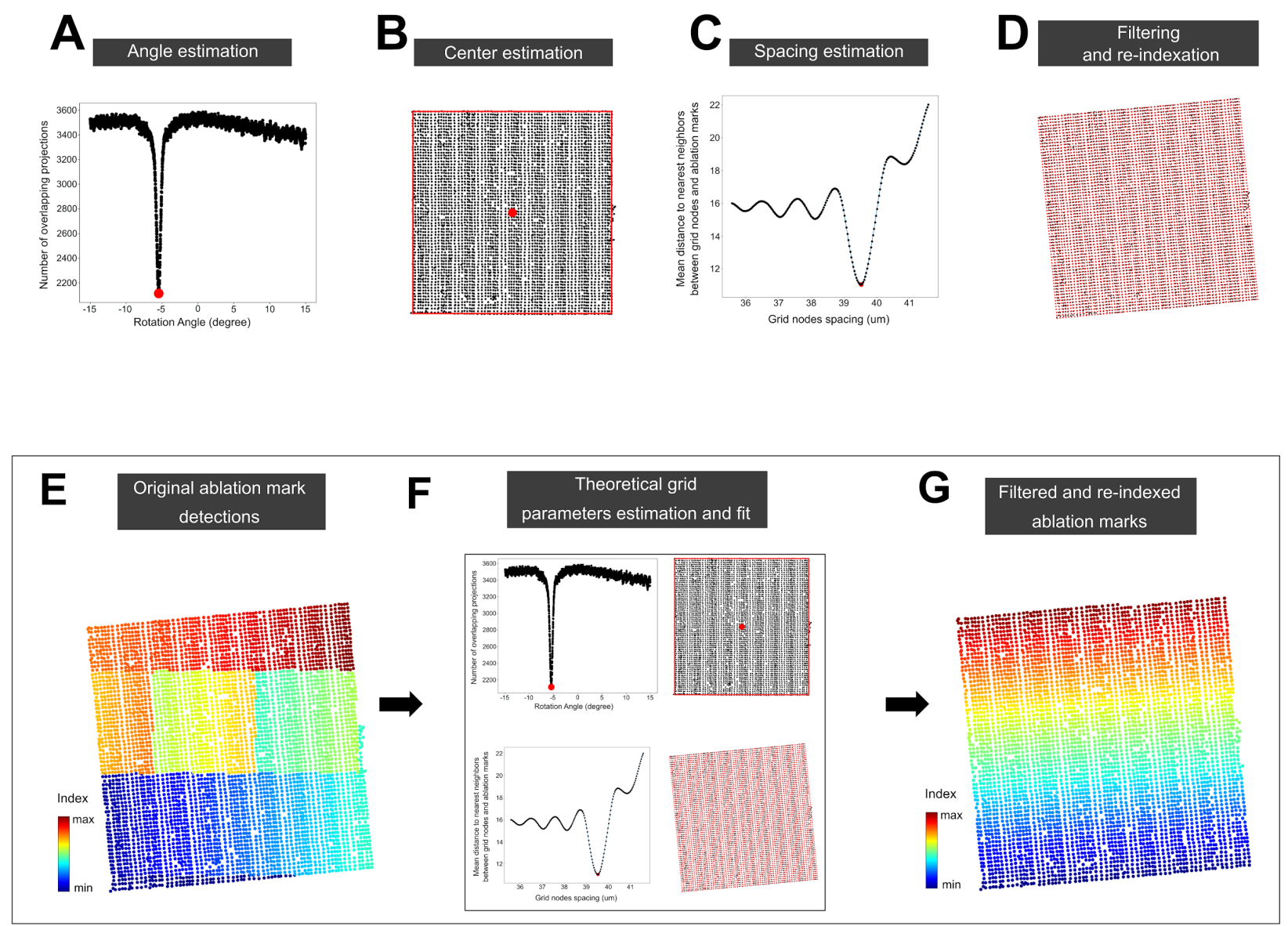

892 Supplementary Figure S10. Illustration of the procedure for fitting a theoretical rectangular

893 grid to the ablation marks segmented in the post-MALDI microscopy images. The three

894 parameters of the grid are estimated using the ablation marks coordinates. In A, the angle is

895 estimated by counting the number of non overlapping ablation marks coordinates projections of

896 the $\mathrm{X}$ axis for different rotation angle. The minimum number of projection is reached for an

897 alignment angle with the projection axis. In B, the center of the grid is estimated from the

898 extrema of the ablation mark coordinates. The spacing of the grid nodes is estimated in $\mathrm{C}$ by

899 measuring the mean distance to the nearest ablation mark to each grid node. The chosen grid

900 node spacing is leads to smallest mean distance to the ablation mark coordinates. The re-

901 indexing in $\mathrm{D}$ is done by choosing the closest ablation mark coordinates from the grid nodes

902 constructed using the parameters defined before (the grid nodes are shown in red, their nearest 
903 ablation mark coordinates are shown in black). In E, the ablation mark coordinates are color

904 coded by their index. An illustration of the different steps for fitting a grid onto the ablation mark

905 coordinates as well as the re-indexing is shown in F. In G, the re-indexed ablation mark are

906 shown. and re-indexing them to associate each detected ablation mark with a MALDI spectrum.

907

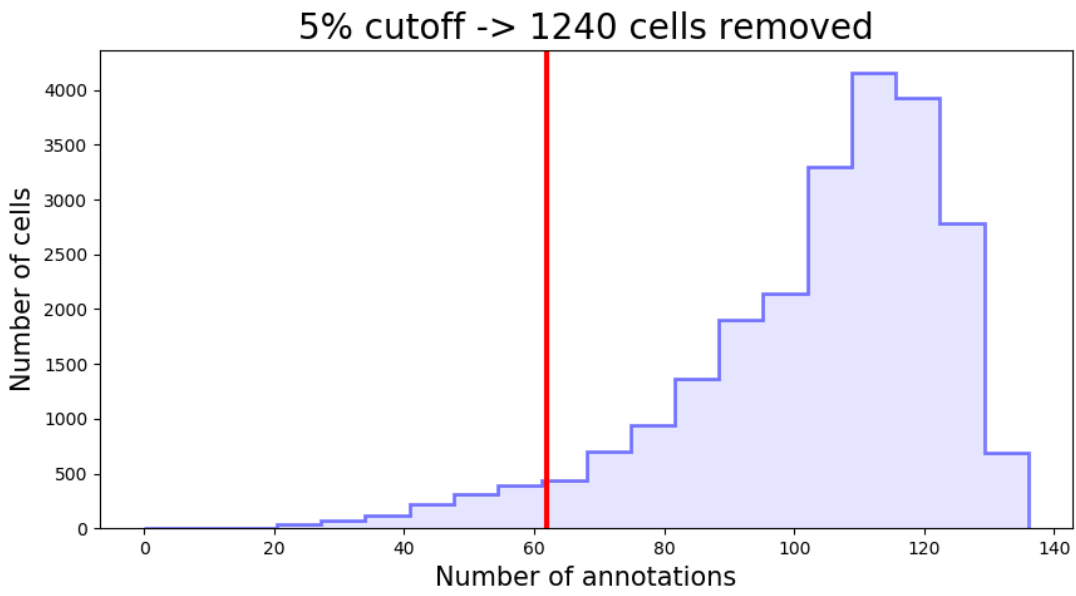

909 Supplementary Figure S11. Filtering out poor quality cells for the dHepaRG experiment

910 (Figure 4). The cells with low number of METASPACE annotations (less than the 5\% lower

911 percentile of the distribution) were removed. 


\section{Supplementary Table}

\begin{tabular}{|c|c|c|c|c|c|c|}
\cline { 2 - 7 } & \multicolumn{3}{|c|}{ Matrix application } & \multicolumn{3}{c|}{ MALDI-imaging } \\
\cline { 2 - 7 } $\begin{array}{c}\text { Sample } \\
\text { (“Condition”_ } \\
\text { "Replicate } \\
\text { number") }\end{array}$ & Date; Time & $\begin{array}{c}\text { Room } \\
\text { temperature } \\
\left({ }^{\circ} \mathrm{C}\right)\end{array}$ & $\begin{array}{c}\text { Room } \\
\text { humidity } \\
(\%)\end{array}$ & Date; Time & $\begin{array}{c}\text { Room } \\
\text { temperature } \\
\left({ }^{\circ} \mathrm{C}\right)\end{array}$ & $\begin{array}{c}\text { Room } \\
\text { humidity } \\
(\%)\end{array}$ \\
\hline FA_1 & $22.06 .17 ; 1.40 \mathrm{pm}$ & 23.1 & 58 & $22.06 .17 ; 2.01 \mathrm{pm}$ & 23.1 & 50 \\
\hline LPS_1 & $22.06 .17 ; 4.10 \mathrm{pm}$ & 23.2 & 60 & $22.06 .17 ; 4.30 \mathrm{pm}$ & 23.7 & 49 \\
\hline FA_2 & $24.06 .17 ; 12.40 \mathrm{am}$ & 21.9 & 52 & $24.06 .17 ; 1.00 \mathrm{pm}$ & 23.3 & 47 \\
\hline LPS_2 & $24.06 .17 ; 3.50 \mathrm{pm}$ & 21.9 & 53 & $24.06 .17 ; 4.10 \mathrm{pm}$ & 23.5 & 47 \\
\hline Untreated_1 & $25.06 .17 ; 6.30 \mathrm{am}$ & 21.9 & 52 & $25.06 .17 ; 7.00 \mathrm{am}$ & 23 & 48 \\
\hline TNFa_1 & $25.06 .17 ; 12.15 \mathrm{am}$ & 22.1 & 60 & $25.06 .17 ; 3.30 \mathrm{pm}$ & 23.6 & 51 \\
\hline FA_3 & $25.06 .17 ; 6.30 \mathrm{am}$ & 21.9 & 58 & $25.06 .17 ; 10.00 \mathrm{am}$ & 23.1 & 47 \\
\hline TNFa_2 & $25.06 .17 ; 9.15 \mathrm{am}$ & 21.9 & 60 & $25.06 .17 ; 9.35 \mathrm{am}$ & 23.1 & 52 \\
\hline Untreated_2 & $25.06 .17 ; 12.41 \mathrm{pm}$ & 21.5 & 55 & $25.06 .17 ; 4.00 \mathrm{pm}$ & 23.1 & 47 \\
\hline Untreated_3 & $25.06 .17 ; 12.41 \mathrm{pm}$ & 21.5 & 55 & $25.06 .17 ; 1.00 \mathrm{pm}$ & 23.1 & 46 \\
\hline LPS_3 & $28.06 .17 ; 2.59 \mathrm{pm}$ & 21.1 & 63 & $28.06 .17 ; 6.30 \mathrm{pm}$ & 23.3 & 48 \\
\hline TNFa_3 & $30.06 .17 ; 2.30 \mathrm{pm}$ & 21.1 & 50 & $30.06 .17 ; 6.41 \mathrm{pm}$ & 22.6 & 44 \\
\hline
\end{tabular}

917 Supplementary Table S1. Experimental design and ambient conditions for matrix application

918 and MALDI-imaging analysis of dHepaRG samples. 


\section{Supplementary Data (attached as a separate file)}

921 Supplementary Data S1. LC-MS/MS validation of METASPACE metabolite annotations.

922 Summary and detailed information about LC-MS/MS validation of METASPACE annotations

923 including MS/MS and chromatographic information. 